\title{
Parametrized Variational Inequality Approaches to Generalized Nash Equilibrium Problems with Shared Constraints ${ }^{1}$
}

\author{
Koichi Nabetani, ${ }^{2}$ Paul Tseng ${ }^{3}$ and Masao Fukushima ${ }^{4}$
}

October 11, 2008; revised April 14, 2009

\begin{abstract}
We consider the generalized Nash equilibrium problem (GNEP), in which each player's strategy set may depend on the rivals' strategies through shared constraints. A practical approach to solving this problem that has received increasing attention lately entails solving a related variational inequality (VI). From the viewpoint of game theory, it is important to find as many GNEs as possible, if not all of them. We propose two types of parametrized VIs related to the GNEP, one pricedirected and the other resource-directed. We show that these parametrized VIs inherit the monotonicity properties of the original VI and, under mild constraint qualifications, their solutions yield all GNEs. We propose strategies to sample in the parameter spaces and show, through numerical experiments on benchmark examples, that the GNEs found by the parametrized VI approaches are widely distributed over the GNE set.
\end{abstract}

Key words. Generalized Nash equilibrium, variational inequality, Karush-KuhnTucker condition, Lagrange multiplier, price-directed parametrizations, resource-directed parametrizations.

\section{Introduction}

The generalized Nash equilibrium problem (GNEP) is a generalization of the standard Nash equilibrium problem (NEP), in which each player's strategy set may depend on the rivals' strategies $[1,13,29]$. Recently, the GNEP has attracted growing attention $[8,20]$ because there are many interesting applications in the fields of economics, mathematics and engineering. For example, Robinson [25, 26] discussed a two-sided game model of combat as an application of GNEP. Wei and Smeers [32] and Hobbs [16] formulated oligopolistic electricity models as GNEPs.

It is well known that NEP where each player solves a convex programming problem can be formulated as a finite-dimensional variational inequality (VI) [9, 14]. The VI has a long history and many solution methods have been proposed; see, e.g., the monograph [9]. On the other hand, GNEP can be formulated as a quasi-variational inequality (QVI) $[13,24]$. However, unlike the VI, there are only few methods available for solving a QVI efficiently [23, 24].

\footnotetext{
${ }^{1}$ This research is supported in part by Grant-in-Aid for Scientific Research from Japan Society for the Promotion of Science, and by National Science Foundation grant DMS-0511283.

${ }^{2}$ Research and Development Headquarters NTT DATA CORPORATION, Toyosu Center Building Annex 3-3-9 Toyosu Koto-ku Tokyo Japan (nabetanik@nttdata.co.jp).

${ }^{3}$ Department of Mathematics, University of Washington, Seattle, WA 98195, USA (tseng@math. washington.edu).

${ }^{4}$ Department of Applied Mathematics and Physics, Graduate School of Informatics, Kyoto University, Kyoto 606-8501, Japan (fuku@i.kyoto-u.ac.jp).
} 
Pang and Fukushima [24] proposed a penalty method for GNEP, which solves a sequence of penalized NEPs. Facchinei and Pang [10] proposed an exact penalty method for GNEP. Fukushima [12] proposed another penalty method for finding a particular GNE called a restricted GNE.

A special class of GNEPs that has received increasing attention lately is that of "shared constraints", which means the constraint functions that depend on rivals' strategies are identical among all players. A solution of such a GNEP can be found via a VI rather than solving a QVI directly [6, 7, 32]. Specifically, Wei and Smeers [32] formulated an oligopolistic electricity model as a GNEP with shared constraints, presented a VI formulation whose solution is a GNE, and established the uniqueness of GNE under some restrictive assumptions. Facchinei et al. [7, Subsection 3.2] also studied a GNEP with shared constraints and proposed to apply a semismooth Newton method to solve its VI formulation. This approach seems promising because we can find a GNE by solving a single VI. However, the GNE found is special in that the multipliers for the shared constraints are identical for all players; also see [6]. Such a GNE is an example of a normalized equilibrium introduced by Rosen [29]; also see Subsection 3.3. In general, GNEP can have multiple, or even infinitely many, solutions [13]; also see Subsection 4.3. In fact, this may be common since the players usually have different objective functions, so the multipliers for the shared constraints need not be equal. In such a case, the VI formulation considered in [6, 32] and [7, Subsection 3.2] may fail to find some important GNEs. The Levenberg-Marquardt-type method proposed in [7, Subsection 3.3] can find other GNEs, but the GNEs must satisfy strict complementarity and local convergence requires a local error bound to hold.

Another approach to solving GNEP involves minimizing the Nikaido-Isoda function [22] using descent methods [15, 19, 20, 29, 30]. Rosen [29] proposed a gradient method to minimize a weighted Nikaido-Isoda-type function. Uryasev and Krawczyk [19, 30] proposed a relaxation method and established its global convergence under a weak convexity-concavity assumption and assumptions on a certain residual term that are not easily verified; also see [20] and references therein. Very recently, von Heusinger and Kanzow [15] proposed a regularization of the Nikaido-Isoda function and reformulated a GNEP with shared constraints as a smooth optimization problem. See [8] for a survey of these and other methods for solving GNEP.

From the practical viewpoint of game theory, it is important to find, if not all, then a set of widely distributed GNEs to convey the possible outcomes [31, Section 15.7]. To our knowledge, no practical method had been previously proposed to achieve this. The aforementioned approaches typically find only one GNE. In this paper we propose two approaches to finding such GNEs in the case of shared constraints. These approaches are based on parametrized VIs, one price-directed and the other resourcedirected, whose solutions include all GNEs. Both approaches extend the VI approaches studied in $[6,7,32]$, and they complement each other by sampling GNEs in different faces of the feasible set.

The paper is organized as follows. In the next section, we recall some definitions and basic concepts. In Section 3, we describe the price-directed and resource-directed parametrized VIs, which are in some sense dual to each other, and derive necessary and sufficient conditions for the solution of these VIs to be GNEs. We also relate the solutions of the price-directed parametrized VIs to normalized equilibria [29], which 
may be viewed as the solutions of a restricted parametrized family of VIs involving weights on the players' objective functions. Sampling strategies in the parameter space are discussed. In Section 4, we describe implementation of the proposed approaches and report some promising numerical results on three benchmark GNEPs. We conclude with some remarks in Section 5.

We use the following notations throughout the paper. For a nonempty closed convex set $X \subseteq \Re^{n}, N_{X}(x)=\left\{d \in \Re^{n} \mid d^{T}(y-x) \leq 0 \forall y \in X\right\}$ denotes the normal cone to $X$ at $x \in X$. For a function $f: \Re^{n} \times \Re^{m} \rightarrow \Re, f(x, \cdot): \Re^{m} \rightarrow \Re$ denotes the function with $x$ being fixed. We denote the non-negative and positive orthants in $\Re^{n}$ by $\Re_{+}^{n}$ and $\Re_{++}^{n}$, respectively, that is,

$$
\Re_{+}^{n}:=\left\{x \in \Re^{n} \mid x \geq 0\right\} \text { and } \Re_{++}^{n}:=\left\{x \in \Re^{n} \mid x>0\right\} .
$$

For vectors $x, y \in \Re^{n},\langle x, y\rangle$ denotes the inner product defined by $\langle x, y\rangle:=x^{T} y$ and $x \perp y$ means $\langle x, y\rangle=0$. For a vector $x,\|x\|$ denotes the Euclidean norm defined by $\|x\|:=\sqrt{\langle x, x\rangle}$. A mapping $F: \Re^{n} \rightarrow \Re^{n}$ is said to be monotone on a nonempty closed convex set $X \subseteq \Re^{n}$ if

$$
\langle F(x)-F(y), x-y\rangle \geq 0 \quad \forall x, y \in X
$$

strictly monotone on $X$ if the above inequality is strict whenever $x \neq y$, and strongly monotone on $X$ if zero on the right-hand side of the above inequality is replaced by $\alpha\|x-y\|^{2}$ for some constant $\alpha>0$.

\section{Problem Formulation and Assumptions}

The generalized Nash game with $N$ players is to find a profile of strategies such that each player's strategy is an optimal response to the rival players' strategies, where each player's strategy set may depend on the rival players' strategies. For $\nu=1, \ldots, N$, let $x_{\nu} \in \Re^{n_{\nu}}$ be a player $\nu$ 's strategy, where $n_{\nu}$ is a positive integer. The vector formed by all these strategies is denoted $x:=\left(x_{\nu}\right)_{\nu=1}^{N} \in \Re^{n}$, where $n:=\sum_{\nu=1}^{N} n_{\nu}$, and the vector formed by all the players' strategies except those of player $\nu$ is denoted $x_{-\nu}:=\left(x_{\nu^{\prime}}\right)_{\nu^{\prime}=1, \nu^{\prime} \neq \nu}^{N} \in \Re^{n_{-\nu}}$, where $n_{-\nu}:=n-n_{\nu}$. For $\nu=1, \ldots, N$, let $K_{\nu}$ be a given point-to-set mapping from $\Re^{n_{-\nu}}$ to $\Re^{n_{\nu}}$. Thus, for each fixed $x_{-\nu}, K_{\nu}\left(x_{-\nu}\right)$ is a subset of $\Re^{n_{\nu}}$, which is the strategy set of player $\nu$ with the other players' strategies given by $x_{-\nu}$.

Each player $\nu=1, \ldots, N$, taking the other players' strategies $x_{-\nu}$ as exogenous variables, solves the minimization problem:

$$
\begin{array}{lll}
P_{\nu}\left(x_{-\nu}\right): & \text { minimize }_{x_{\nu}} & \theta_{\nu}\left(x_{\nu}, x_{-\nu}\right) \\
& \text { subject to } \quad x_{\nu} \in K_{\nu}\left(x_{-\nu}\right),
\end{array}
$$

where $\theta_{\nu}: \Re^{n} \rightarrow \Re$ is a given cost function of player $\nu$. A vector $x=\left(x_{\nu}\right)_{\nu=1}^{N}$ is said to be feasible to GNEP if $x_{\nu} \in K_{\nu}\left(x_{-\nu}\right)$ for each $\nu=1, \ldots, N$.

The GNEP is to find a vector $x^{*}=\left(x_{\nu}^{*}\right)_{\nu=1}^{N} \in \Re^{n}$ such that

$$
x_{\nu}^{*} \text { is an optimal solution of } P_{\nu}\left(x_{-\nu}^{*}\right) \text { for all } \nu=1, \ldots, N \text {. }
$$


A vector $x^{*}$ satisfying (2) is called a generalized Nash equilibrium (GNE). The set of GNEs is denoted by SOL ${ }^{\text {GNEP }}$.

In many practical applications, the strategy set $K_{\nu}\left(x_{-\nu}\right)$ of player $\nu$ is represented by finitely many inequality constraints. We will consider the important case of "shared constraints", that is, all players share joint constraints that depend on all the players' strategies. Specifically, we assume that the feasible strategy set $K_{\nu}\left(x_{-\nu}\right)$ of player $\nu$ has the form

$$
K_{\nu}\left(x_{-\nu}\right)=\left\{x_{\nu} \in X_{\nu} \mid g(x) \leq 0\right\}
$$

where $g=\left(g_{i}\right)_{i=1}^{m}: \Re^{n} \rightarrow \Re^{m}$ and $X_{\nu} \subseteq \Re^{n_{\nu}}$, with a positive integer $m$. Thus, player $\nu$ 's strategy is constrained in two ways; joint constraints that depend also on the other players' strategies, i.e., $g(x) \leq 0$, and individual constraints that depend only on player $\nu$ 's strategy, i.e., $x_{\nu} \in X_{\nu}$. (A more general model involves individual joint constraints $g_{\nu}(x) \leq 0$ for each player $\nu$ instead of shared constraints. It is an open question whether our results can be extended to this more general model.)

In what follows, we let

$$
X_{-\nu}:=\prod_{\substack{\nu^{\prime}=1 \\ \nu^{\prime} \neq \nu}}^{N} X_{\nu^{\prime}} .
$$

We distinguish these two types of constraints since our parametrization will involve only the joint constraints. Throughout this paper, we make the following blanket assumption on the smoothness and convexity of functions involved in the GNEP.

Assumption A. For $\nu=1, \ldots, N$, the set $X_{\nu}$ is nonempty, closed, convex, and, for each fixed $x_{-\nu} \in X_{-\nu}$, the function $\theta_{\nu}\left(\cdot, x_{-\nu}\right)$ is differentiable and convex. Also, $g_{1}, \ldots, g_{m}$ are differentiable and convex.

Assumption A is reasonable and is satisfied by Harker's example [13], a river basin pollution game [19, Section 5.3], electricity market models [4, Section IV.B], [16], [24, Section 5.3], [32, Section 1], and an internet switching model [6, Section 5]. By Assumption A, problem (1) is a differentiable convex programming problem. Thus a necessary and sufficient condition for $x_{\nu}^{*} \in K_{\nu}\left(x_{-\nu}^{*}\right)$ to be optimal for (1) is that the inequalities

$$
\left\langle\nabla_{x_{\nu}} \theta_{\nu}\left(x_{\nu}^{*}, x_{-\nu}^{*}\right), x_{\nu}-x_{\nu}^{*}\right\rangle \geq 0 \quad \forall x_{\nu} \in K_{\nu}\left(x_{-\nu}^{*}\right)
$$

hold. Thus, by defining

$$
\begin{aligned}
F(x) & :=\left(\nabla_{x_{\nu}} \theta_{\nu}(x)\right)_{\nu=1}^{N}, \\
K(x) & :=\prod_{\nu=1}^{N} K_{\nu}\left(x_{-\nu}\right),
\end{aligned}
$$

it follows that $x^{*}$ is a GNE if and only if $x^{*} \in K\left(x^{*}\right)$ and

$$
\left\langle F\left(x^{*}\right), x-x^{*}\right\rangle \geq 0 \quad \forall x \in K\left(x^{*}\right) .
$$

The latter problem is a QVI. 
Suppose that $x^{*}$ is a solution of GNEP. Then, for each $\nu=1, \ldots, N, x_{\nu}^{*}$ is an optimal solution of the convex programming problem:

$$
\begin{array}{lll}
P_{\nu}\left(x_{-\nu}^{*}\right): & \text { minimize }_{x_{\nu}} & \theta_{\nu}\left(x_{\nu}, x_{-\nu}^{*}\right) \\
& \text { subject to } \quad g\left(x_{\nu}, x_{-\nu}^{*}\right) \leq 0, \quad x_{\nu} \in X_{\nu} .
\end{array}
$$

Under a suitable CQ at $x^{*}$ (see, e.g., [2, Section 5.4], [28]), there exists for each $\nu=$ $1, \ldots, N$ a vector $\lambda_{\nu}^{*} \in \Re^{m}$ satisfying the Karush-Kuhn-Tucker (KKT) condition:

$$
\begin{gathered}
0 \in \nabla_{x_{\nu}} L_{\nu}\left(x_{\nu}, x_{-\nu}^{*}, \lambda_{\nu}\right)+N_{X_{\nu}}\left(x_{\nu}\right), \\
0 \leq \lambda_{\nu} \perp g\left(x_{\nu}, x_{-\nu}^{*}\right) \leq 0, \quad x_{\nu} \in X_{\nu},
\end{gathered}
$$

where the Lagrangian function $L_{\nu}$ is defined by

$$
L_{\nu}\left(x, \lambda_{\nu}\right):=\theta_{\nu}(x)+\left\langle g(x), \lambda_{\nu}\right\rangle .
$$

The vector $\lambda^{*}=\left(\lambda_{\nu}^{*}\right)_{\nu=1}^{N} \in \Re^{N m}$ is called a Lagrange multiplier vector. Under Assumption A, if $\left(x^{*}, \lambda^{*}\right)$ satisfies (5), then $x^{*}$ is a GNE. A well-known CQ at $x$ is the Mangasarian-Fromovitz CQ (MFCQ) [28, page 198]: For $\nu=1, \ldots, N$,

$$
\left\{\begin{array}{c}
0 \in \nabla_{x_{\nu}} g(x) \lambda_{\nu}+N_{X_{\nu}}\left(x_{\nu}\right) \\
0 \leq \lambda_{\nu} \perp g(x) \leq 0, \quad x_{\nu} \in X_{\nu}
\end{array}\right\} \quad \Longrightarrow \quad \lambda_{\nu}=0
$$

where

$$
\nabla_{x_{\nu}} g(x):=\left(\nabla_{x_{\nu}} g_{1}(x), \cdots, \nabla_{x_{\nu}} g_{m}(x)\right) .
$$

Another useful CQ at $x$ is the Linear Independence CQ (LICQ): For $\nu=1, \ldots, N$,

$$
\left\{\begin{array}{c}
0 \in \nabla_{x_{\nu}} g(x) \lambda_{\nu}+N_{X_{\nu}}\left(x_{\nu}\right)+\left(-N_{X_{\nu}}\left(x_{\nu}\right)\right), \\
\lambda_{\nu} \perp g\left(x^{*}\right) \leq 0, \quad x_{\nu} \in X_{\nu}
\end{array}\right\} \quad \Longrightarrow \quad \lambda_{\nu}=0 .
$$

This CQ implies uniqueness of the multiplier vector $\lambda^{*}$ for each $x^{*}$.

\section{Parameterized VI Approaches to GNE}

In this section, we propose two approaches, based on parametrized VI, for finding all GNEs.

We begin by noting that $\left(x^{*}, \lambda^{*}\right) \in \Re^{n} \times \Re^{N m}$ satisfies the KKT condition (5) if and only if $\left(x^{*}, \lambda^{*}\right)$ satisfies

$$
\begin{gathered}
0 \in \nabla_{x_{\nu}} \theta_{\nu}(x)+\nabla_{x_{\nu}} g(x) \lambda_{\nu}+N_{X_{\nu}}\left(x_{\nu}\right), \quad \nu=1, \ldots, N, \\
0 \leq \lambda_{\nu} \perp g(x) \leq 0, \quad x_{\nu} \in X_{\nu}, \quad \nu=1, \ldots, N .
\end{gathered}
$$

Also note from the complementarity condition that $g_{i}(x)<0$ implies that $\lambda_{\nu, i}=0$ for all $\nu=1, \ldots, N$ and $\lambda_{\nu, i}>0$ for some $\nu$ implies that $g_{i}(x)=0$.

The VI approach to finding a GNE $[6,7,32]$ is to define

$$
X:=\left\{x \in \Re^{n} \mid g(x) \leq 0\right\} \cap \prod_{\nu=1}^{N} X_{\nu},
$$


which is a closed convex set, and solve the following $\mathrm{VI}(F, X)$ :

Find $x^{*} \in X \quad$ such that $\left\langle F\left(x^{*}\right), x-x^{*}\right\rangle \geq 0 \quad \forall x \in X$,

where $F: \Re^{n} \rightarrow \Re^{n}$ is defined by (4). The solution set of $\operatorname{VI}(F, X)$ is denoted by $\operatorname{SOL}(F, X)^{5}$. A vector $x^{*}$ belongs to $\operatorname{SOL}(F, X)$ if and only if it is an optimal solution of the convex programming problem:

$$
\begin{array}{ll}
\operatorname{minimize}_{x} & \left\langle F\left(x^{*}\right), x\right\rangle \\
\text { subject to } & x \in X,
\end{array}
$$

whose KKT condition is a special case of (7) with $\lambda_{1}=\cdots=\lambda_{N}$. Specifically, the following result is known [7, Theorem 3.6] (also see [32, Theorem 2] and [6, Theorem 2.1]).

Theorem 3.1. Every $x^{*} \in \operatorname{SOL}(F, X)$ is a GNE. Furthermore, if $x^{*}$ together with some Lagrange multiplier vector $\bar{\lambda} \in \Re^{m}$ satisfies the KKT condition for (8), then $x^{*}$ and $\lambda^{*}=\left(\lambda_{\nu}^{*}\right)_{\nu=1}^{N}$ with $\lambda_{1}^{*}=\cdots=\lambda_{N}^{*}=\bar{\lambda}$ satisfy $(7)$.

In many practical situations, since the players have different objective functions as well as their own constraints, the multipliers for the shared constraints in GNEP may not be identical. Therefore, in general, there would be many GNEs that are not solutions of $\mathrm{VI}(F, X)$. This is illustrated in the following example.

Example 1. Consider the two-person game, where the problems of player 1 and player 2 are defined by

$$
\begin{array}{lll}
P_{1}\left(x_{2}\right): & \operatorname{minimize}_{x_{1}} & x_{1}^{2}-x_{1} x_{2}-x_{1} \\
& \text { subject to } & x_{1} \geq 0 \\
& x_{1}+x_{2} \leq 1
\end{array}
$$

and

$$
\begin{aligned}
& P_{2}\left(x_{1}\right): \operatorname{minimize}_{x_{2}} \quad x_{2}^{2}-\frac{1}{2} x_{1} x_{2}-2 x_{2} \\
& \text { subject to } x_{2} \geq 0 \\
& x_{1}+x_{2} \leq 1 \text {, }
\end{aligned}
$$

respectively. The set of GNEs consists of infinitely many vectors

$$
\left(\begin{array}{l}
x_{1} \\
x_{2}
\end{array}\right)=\left(\begin{array}{c}
t \\
1-t
\end{array}\right), \quad 0 \leq t \leq \frac{2}{3}
$$

On the other hand, the corresponding $F: \Re^{2} \rightarrow \Re^{2}$ and $X \subseteq \Re^{2}$ are given by

$$
F(x)=\left(\begin{array}{c}
2 x_{1}-x_{2}-1 \\
-\frac{1}{2} x_{1}+2 x_{2}-2
\end{array}\right), \quad X=\left\{x \in \Re_{+}^{2} \mid x_{1}+x_{2} \leq 1\right\},
$$

and the solution of $\mathrm{VI}(F, X)$ is uniquely given by $x=\left(\frac{4}{11}, \frac{7}{11}\right)^{T}$.

In this example, the mapping $F$ is strongly monotone and hence $\operatorname{SOL}(F, X)$ is a singleton [9], but there are infinitely many GNEs. Uniqueness of GNE requires restrictive assumptions $[13,32]$ which cannot be expected to hold in most applications. In general, the above VI approach can find only a part of the GNEs.

\footnotetext{
${ }^{5} \mathrm{~A}$ solution of $\mathrm{VI}(F, X)$ is called a variational equilibrium in [8] and is shown to be a particular instance of a normalized equilibrium introduced by Rosen [29]. In Section 3.3, we will discuss the relationship between normalized equilibria and solutions of the parametrized VI.
} 


\subsection{Price-Directed Parametrization}

Now, we construct a family of VIs that contains $\mathrm{VI}(F, X)$ as a particular instance. Let $\omega=\left(\omega_{\nu}\right)_{\nu=1}^{N} \in \Re_{+}^{N m}$, with $\omega_{\nu} \in \Re_{+}^{m}, \nu=1, \ldots, N$, be a vector of parameters. Define the function $F^{\omega}: \Re^{n} \rightarrow \Re^{n}$ by

$$
F^{\omega}(x):=\left(\nabla_{x_{\nu}} \theta_{\nu}(x)+\nabla_{x_{\nu}} g(x) \omega_{\nu}\right)_{\nu=1}^{N} .
$$

Note that $\mathrm{VI}\left(F^{\omega}, X\right)$ reduces to $\mathrm{VI}(F, X)$ if the parameter $\omega$ is set to zero. Moreover, just as VI $(F, X)$ corresponds to the GNEP (1) with the feasible strategy sets given by (3), VI $\left(F^{\omega}, X\right)$ corresponds to the parametrized GNEP in which player $\nu$ 's optimization problem is given by

$$
\begin{array}{ll}
\operatorname{minimize}_{x_{\nu}} & \theta_{\nu}\left(x_{\nu}, x_{-\nu}\right)+\left\langle g\left(x_{\nu}, x_{-\nu}\right), \omega_{\nu}\right\rangle \\
\text { subject to } & g\left(x_{\nu}, x_{-\nu}\right) \leq 0, \quad x_{\nu} \in X_{\nu} .
\end{array}
$$

Here $\omega_{\nu}$ may be viewed as unit prices that player $\nu$ pays for the shared resources. The following proposition shows that, under appropriate assumptions, the monotonicity of $F$ implies the monotonicity of $F^{\omega}$ for any $\omega \in \Re_{+}^{N m}$.

Proposition 3.1. Assume that $F$ is monotone (strictly monotone, strongly monotone) on $X$. Assume further that the shared constraint function $g$ is separable, that is, $g(x)$ can be written as

$$
g(x)=\sum_{\nu=1}^{N} g_{\nu}\left(x_{\nu}\right),
$$

where $g_{\nu}=\left(g_{\nu, i}\right)_{i=1}^{m}: \Re^{n_{\nu}} \rightarrow \Re^{m}, \nu=1, \ldots, N$, are differentiable convex functions. Then, for any $\omega \in \Re_{+}^{N m}$, $F^{\omega}$ is also monotone (strictly monotone, strongly monotone) on $X$.

Proof. Since $g$ is separable, we have

$$
F^{\omega}(x)=F(x)+\left(\nabla g_{\nu}\left(x_{\nu}\right) \omega_{\nu}\right)_{\nu=1}^{N} .
$$

For each $\nu=1, \ldots, N$ and $i=1, \ldots, m, \nabla g_{\nu, i}$ is monotone on $X_{\nu}$ since $g_{\nu, i}$ is a differentiable convex function. Therefore $F^{\omega}$ is monotone (strictly monotone, strongly monotone) on $X$ for any $\omega \in \Re_{+}^{N m}$.

Under the assumptions of this proposition, we can apply, for example, Newtontype or projection-type methods to solve VI $\left(F^{\omega}, X\right)$; see [9] and references therein. A sufficient condition for $F$ to be strictly monotone in the setting of spatial oligopolistic electricity models is given in [32, Theorems 5 and 6$]$.

We now investigate the relationship between $\mathrm{VI}\left(F^{\omega}, X\right)$ and GNEP. The KKT condition for $\mathrm{VI}\left(F^{\omega}, X\right)$ can be written as

$$
\begin{gathered}
0 \in\left(\nabla_{x_{\nu}} \theta_{\nu}(x)+\nabla_{x_{\nu}} g(x) \omega_{\nu}\right)+\nabla_{x_{\nu}} g(x) \pi+N_{X_{\nu}}\left(x_{\nu}\right), \quad \nu=1, \ldots, N, \\
0 \leq \pi \perp g(x) \leq 0, \quad x_{\nu} \in X_{\nu}, \quad \nu=1, \ldots, N .
\end{gathered}
$$

For each GNE $x$, let

$$
\Lambda(x):=\left\{\lambda \in \Re^{N m} \mid(x, \lambda) \text { satisfies the KKT condition }(7)\right\} .
$$

By comparing the KKT condition (11) with (7), we have the following result. 
Theorem 3.2. For any GNE $x^{*}$, if $\lambda^{*} \in \Lambda\left(x^{*}\right)$, then $x^{*} \in \operatorname{SOL}\left(F^{\lambda^{*}}, X\right)$.

Proof. Fix any GNE $x^{*}$, and assume that $\lambda^{*} \in \Lambda\left(x^{*}\right)$. Then $\left(x^{*}, \lambda^{*}\right)$ satisfies the KKT condition (7). This in turn shows that $\left(x^{*}, 0\right)$ satisfies the KKT condition for $\mathrm{VI}\left(F^{\lambda^{*}}, X\right)$ and hence $x^{*}$ is a solution of $\mathrm{VI}\left(F^{\lambda^{*}}, X\right)$.

Corollary 3.1. If $\Lambda\left(x^{*}\right) \neq \emptyset$ for every GNE $x^{*}$, then

$$
\bigcup_{\omega \in \Re_{+}^{N m}} \operatorname{SOL}\left(F^{\omega}, X\right) \supseteq \mathrm{SOL}^{\mathrm{GNEP}} .
$$

For an arbitrary GNE $x^{*}$, let $\lambda^{*} \in \Lambda\left(x^{*}\right)$ and define

$$
\begin{aligned}
\underline{\lambda}_{i}^{*} & :=\min _{\nu=1, \ldots, N} \lambda_{\nu, i}^{*} \quad i=1, \ldots, m \\
\omega_{\nu, i} & :=\lambda_{\nu, i}^{*}-\underline{\lambda}_{i}^{*} \quad i=1, \ldots, m, \nu=1, \ldots, N .
\end{aligned}
$$

Then $x^{*}$ along with $\underline{\lambda}^{*}$ satisfies the KKT condition for $\operatorname{VI}\left(F^{\omega}, X\right)$, and hence $x^{*}$ also belongs to $\operatorname{SOL}\left(F^{\omega}, X\right)$. This implies that for any GNE $x^{*}$ satisfying $\Lambda\left(x^{*}\right) \neq \emptyset$, there always exists a $\omega \in \Re_{+}^{N m}$ such that $x^{*} \in \operatorname{SOL}\left(F^{\omega}, X\right)$ and, for each $i, \omega_{\nu, i}=0$ for some $\nu$. This observation yields the following result that sharpens Corollary 3.1.

Corollary 3.2. If $\Lambda\left(x^{*}\right) \neq \emptyset$ for every GNE $x^{*}$, then

$$
\bigcup_{\omega \in \mathcal{W}} \operatorname{SOL}\left(F^{\omega}, X\right) \supseteq \mathrm{SOL}^{\mathrm{GNEP}},
$$

where the set $\mathcal{W}$ is defined $b y^{6}$

$$
\mathcal{W}:=\prod_{i=1}^{m}\left(\bigcup_{\nu=1}^{N}\left\{\omega_{i} \in \Re_{+}^{N} \mid \omega_{\nu, i}=0\right\}\right) \subseteq \Re_{+}^{N m} .
$$

This corollary suggests that, for finding GNEs, it suffices to restrict $\omega$ to the parameter set $\mathcal{W}$ instead of $\Re_{+}^{N m}$.

In general, a solution of $\mathrm{VI}\left(F^{\omega}, X\right)$ for some $\omega \in \Re_{+}^{m}$ need not be a GNE. To see this, let us consider the $\operatorname{VI}\left(F^{\omega}, X\right)$ in Example 1 with $\omega=(1,1)^{T}$. Then

$$
F^{\omega}(x)=\left(\begin{array}{c}
2 x_{1}-x_{2} \\
-\frac{1}{2} x_{1}+2 x_{2}-1
\end{array}\right)
$$

and $\operatorname{VI}\left(F^{\omega}, X\right)$ has the unique solution $x=\left(\frac{2}{7}, \frac{4}{7}\right)^{T}$ which is not a GNE.

The next result gives a sufficient condition for a solution of $\mathrm{VI}\left(F^{\omega}, X\right)$ to be a GNE.

Theorem 3.3. For any $\omega \in \Re_{+}^{N m}$ and any $\left(x^{*}, \pi^{*}\right) \in \Re^{n} \times \Re^{m}$ satisfying the KKT condition (11), a sufficient condition for $x^{*}$ to be a GNE is that

$$
\left\langle g\left(x^{*}\right), \omega_{\nu}\right\rangle=0, \quad \nu=1, \ldots, N .
$$

If in addition the LICQ (6) holds at $x^{*}$, then (14) is also a necessary condition for $x^{*}$ to be a GNE.

\footnotetext{
${ }^{6} \operatorname{In}(13), \omega_{i}$ denotes an $N$-vector $\left(\omega_{1, i}, \ldots, \omega_{N, i}\right)^{T}$, while $\omega_{\nu}$ denotes an $m$-vector $\left(\omega_{\nu, 1}, \ldots, \omega_{\nu, m}\right)^{T}$ in other places. This slight abuse of notation should cause no confusion.
} 
Proof. Let $\lambda^{*}=\left(\lambda_{\nu}^{*}\right)_{\nu=1}^{N} \in \Re^{N m}$ be given by

$$
\lambda_{\nu}^{*}:=\pi^{*}+\omega_{\nu} \geq 0, \quad \nu=1, \ldots, N .
$$

By (11) and (14), we have

$$
\left\langle g\left(x^{*}\right), \lambda_{\nu}^{*}\right\rangle=\left\langle g\left(x^{*}\right), \pi^{*}+\omega_{\nu}\right\rangle=0 .
$$

Hence $\left(x^{*}, \lambda^{*}\right)$ satisfies the KKT condition (7). This shows that $x^{*}$ is a GNE.

Conversely, suppose that $x^{*}$ is a GNE and the LICQ (6) holds at $x^{*}$. By LICQ, the Lagrange multiplier vector $\lambda^{*}$ satisfying (7) with $x=x^{*}$ and the Lagrange multiplier vector $\pi^{*}$ satisfying (11) with $x=x^{*}$ are both unique. Then we must have

$$
\lambda_{\nu}^{*}=\pi^{*}+\omega_{\nu}, \quad \nu=1, \ldots, N
$$

Moreover, $\left\langle g\left(x^{*}\right), \lambda_{\nu}^{*}\right\rangle=0, \nu=1, \ldots, N$ and $\left\langle g\left(x^{*}\right), \pi^{*}\right\rangle=0$, which together yield

$$
\left\langle g\left(x^{*}\right), \omega_{\nu}\right\rangle=\left\langle g\left(x^{*}\right), \lambda_{\nu}^{*}-\pi^{*}\right\rangle=0, \quad \nu=1, \ldots, N .
$$

Remark 3.1. When $\omega=0$, we have $F^{\omega} \equiv F$. Moreover, (14) clearly holds. Hence Theorem 3.3 generalizes Theorem 3.1.

Corollary 3.1 shows that $\mathrm{SOL}{ }^{\mathrm{GNEP}}$ is contained in the union of $\operatorname{SOL}\left(F^{\omega}, X\right)$ over all $\omega \in \Re_{+}^{N m}$. We show below that, under the following sequentially bounded CQ (SBCQ), the range of parameter $\omega$ can be restricted to a bounded set $\Omega \subseteq \Re_{+}^{N m}$ and the union of $\operatorname{SOL}\left(F^{\omega}, X\right)$ still contains an "arbitrarily large" subset of SOL ${ }^{\mathrm{GNEP}}$.

Definition 3.1 (SBCQ). For every bounded sequence $\left\{x^{k}\right\} \subseteq \mathrm{SOL}^{\mathrm{GNEP}}$, there exists a bounded sequence $\left\{\lambda^{k}\right\} \subseteq \Re^{N m}$ satisfying $\lambda^{k} \in \Lambda\left(x^{k}\right)$ for all $k$.

The SBCQ was introduced in the study of mathematical programs with equilibrium constraints (MPEC) [21]. It is a unification of well-known CQs such as MFCQ and the constant rank CQ [17], and plays an important role not only in MPEC but also in GNEP [24]. It can be shown that if the function $g$ is affine and $X_{1}, \ldots, X_{N}$ are polyhedral sets, then SBCQ holds [24].

Theorem 3.4. Assume that $S B C Q$ holds. For any bounded set $\mathcal{E} \subseteq \Re^{n}$, there exists a bounded set $\Omega \subseteq \mathcal{W} \subseteq \Re_{+}^{N m}$ such that

$$
\bigcup_{\omega \in \Omega} \operatorname{SOL}\left(F^{\omega}, X\right) \supseteq \mathcal{E} \cap \mathrm{SOL}^{\mathrm{GNEP}}
$$

where $\mathcal{W}$ is given by (13).

Proof. Fix any bounded set $\mathcal{E} \subseteq \Re^{n}$. We claim that there exists a bounded set $\hat{\Omega} \subseteq \Re_{+}^{N m}$ such that

$$
\Lambda(x) \cap \hat{\Omega} \neq \emptyset \quad \forall x \in \mathcal{E} \cap \mathrm{SOL}^{\mathrm{GNEP}} .
$$

If this were not true, then there would exist a sequence $\left\{x^{k}\right\} \subseteq \mathcal{E} \cap \mathrm{SOL}{ }^{\mathrm{GNEP}}$ such that $\min _{\lambda \in \Lambda\left(x^{k}\right)}\|\lambda\| \rightarrow \infty$. (Here we use the convention that $\min _{\lambda \in \Lambda\left(x^{k}\right)}\|\lambda\|=\infty$ whenever 
$\Lambda\left(x^{k}\right)=\emptyset$.) However, since $\left\{x^{k}\right\}$ lies in a bounded set $\mathcal{E}$, SBCQ would imply that $\min _{\lambda \in \Lambda\left(x^{k}\right)}\|\lambda\|$ is bounded, a contradiction. We assume without loss of generality that $\hat{\Omega}$ is a box, i.e., a Cartesian product of intervals, which contains the origin.

Fix any $x^{*} \in \mathcal{E} \cap \mathrm{SOL}^{\mathrm{GNEP}}$. By (15), there exists $\lambda^{*} \in \Lambda\left(x^{*}\right) \cap \hat{\Omega}$. By Theorem 3.2, $x^{*} \in \operatorname{SOL}\left(F^{\lambda^{*}}, X\right)$ and hence $x^{*} \in \operatorname{SOL}\left(F^{\omega}, X\right)$, where $\omega$ is given by (12). Moreover, $\omega \in \mathcal{W}$ and $0 \leq \omega \leq \lambda^{*}$. Since $\lambda^{*} \in \hat{\Omega}$ and $\hat{\Omega}$ is a box containing the origin, the latter implies $\omega \in \hat{\Omega}$. Thus every element of $\mathcal{E} \cap \operatorname{SOL}{ }^{\text {GNEP }}$ belongs to $\operatorname{SOL}\left(F^{\omega}, X\right)$ for some $\omega \in \hat{\Omega} \cap \mathcal{W}$. This proves the desired inclusion, with $\Omega:=\hat{\Omega} \cap \mathcal{W}$.

If SOL ${ }^{\text {GNEP }}$ is bounded, then we can take $\mathcal{E}=\mathrm{SOL}^{\mathrm{GNEP}}$. Unfortunately, Theorem 3.4 does not say how large $\Omega$ should be. This is a question for further study.

\subsection{Resource-Directed Parametrization}

In the case where $g$ is affine and $X_{1}, \ldots, X_{N}$ are polyhedral, it is known that $\Lambda(x) \neq \emptyset$ for every GNE $x$. Otherwise, $\Lambda(x)$ could be empty for some GNE $x$, and the pricedirected dual parametrization approach of Section 3.1 would not be able to find this GNE. Such a GNE is of interest since it indicates a high sensitivity of individual costs to allocation of joint resources. Moreover, price-directed parametrization could be inefficient locally in the sense that large changes in $\omega$ would result in small changes in $\operatorname{SOL}\left(F^{\omega}, X\right)$.

In this section, we consider the case of separable $g$ and present a resource-directed primal parametrization approach that does not rely on the existence of a Lagrange multiplier vector. We motivate this with an example.

Example 2. Consider a modification of Example 1 where the shared constraint $x_{1}+$ $x_{2} \leq 1$ is changed to $x_{1}^{2}+x_{2}^{2} \leq 1$. It can be seen that the GNEs are the vectors

$$
\left(\begin{array}{l}
x_{1} \\
x_{2}
\end{array}\right)=\left(\begin{array}{c}
t \\
\sqrt{1-t^{2}}
\end{array}\right), \quad 0 \leq t \leq \frac{4}{5}
$$

The corresponding $\mathrm{VI}(F, X)$ still has only one solution since $F$ is unchanged and remains strongly monotone. At the GNE $x=(0,1)^{T}, \Lambda(x)=\emptyset$ (due to the constraint $x_{1}^{2} \leq 0$ in the problem of player 1 ). Thus the approach of Section 3.1 would not find this GNE, though it is clearly of interest.

The shared constraint function in Example 2 is separable, which we will exploit in developing our primal, or resource-directed, parametrization. Specifically, we make the following blanket assumption throughout this section.

Assumption B. The shared constraint function $g$ has the form (10), where $g_{\nu}=$ $\left(g_{\nu, i}\right)_{i=1}^{m}: \Re^{n_{\nu}} \rightarrow \Re^{m}, \nu=1, \ldots, N$, and each $g_{\nu, i}$ is a differentiable convex function.

Assumption B is reasonable and is satisfied by the GNEPs in [4, Section IV.B], [6, Section 5], [13], [16], [19, Section 5.3], [24, Section 5.3], [32, Section 1], for which $g$ is affine. Now, we construct a family of VIs that contains $\mathrm{VI}(F, X)$ as a particular instance. Let $\beta=\left(\beta_{\nu}\right)_{\nu=1}^{N} \in \Re^{N m}$ with $\beta_{\nu} \in \Re^{m}, \nu=1, \ldots, N$, be a vector of parameters satisfying $\sum_{\nu=1}^{N} \beta_{\nu}=0$. Define the set $X^{\beta} \subseteq X$ by

$$
X^{\beta}:=X_{1}^{\beta_{1}} \times \cdots \times X_{N}^{\beta_{N}} \quad \text { with } \quad X_{\nu}^{\beta_{\nu}}:=\left\{x_{\nu} \in X_{\nu} \mid g_{\nu}\left(x_{\nu}\right) \leq \beta_{\nu}\right\}, \nu=1, \ldots, N,
$$


and consider VI $\left(F, X^{\beta}\right)$. By Assumption $\mathrm{B}, X^{\beta}$ is closed and convex (possibly empty). Intuitively, we parametrize the division of resources among the players, reminiscent of Bender's decomposition.

Now, we investigate the relationship between $\mathrm{VI}\left(F, X^{\beta}\right)$ and GNEP. The following result is easy to see.

Theorem 3.5. For any GNE $x^{*}$, we have $x^{*} \in \operatorname{SOL}\left(F, X^{\beta}\right)$ and $\sum_{\nu=1}^{N} \beta_{\nu}=0$, where we let $\beta_{\nu}=g_{\nu}\left(x_{\nu}^{*}\right)-\alpha_{\nu} g\left(x^{*}\right)$ with arbitrary real numbers $\alpha_{\nu}$ such that $\sum_{\nu=1}^{N} \alpha_{\nu}=1$ and $\alpha_{\nu}>0, \nu=1, \ldots, N$.

\section{Corollary 3.3.}

$$
\bigcup_{\sum_{\nu=1}^{N} \beta_{\nu}=0} \operatorname{SOL}\left(F, X^{\beta}\right) \supseteq \operatorname{SOL}^{\mathrm{GNEP}} .
$$

In Example 2, if we choose $g_{1}\left(x_{1}\right)=x_{1}^{2}$ and $g_{2}\left(x_{2}\right)=x_{2}^{2}-1$, then $\operatorname{SOL}\left(F, X^{\beta}\right)=$ $\left\{(0,1)^{T}\right\}$ for $\beta=(0,0)^{T}$. In general, VI $\left(F, X^{\beta}\right)$ need not have a solution, or a solution need not be a GNE. The next result gives a sufficient condition for a solution of $\mathrm{VI}\left(F, X^{\beta}\right)$ to be a GNE.

Theorem 3.6. For any $\beta \in \Re^{N m}$ with $\sum_{\nu=1}^{N} \beta_{\nu}=0$ and any $x^{*} \in \operatorname{SOL}\left(F, X^{\beta}\right)$, a sufficient condition for $x^{*}$ to be a GNE is that

$$
\text { for each } i=1, \ldots, m, \quad\left\{\begin{array}{lll}
\text { either } & g_{\nu, i}\left(x_{\nu}^{*}\right)=\beta_{\nu, i} & \nu=1, \ldots, N \\
\text { or } & g_{\nu, i}\left(x_{\nu}^{*}\right)<\beta_{\nu, i} & \nu=1, \ldots, N .
\end{array}\right\}
$$

Proof. Since $x^{*}$ is a solution of $\mathrm{VI}\left(F, X^{\beta}\right)$, for each $\nu=1, \ldots, N$, we have that $x_{\nu}^{*}$ is a solution of $\mathrm{VI}\left(\nabla_{x_{\nu}} \theta_{\nu}\left(\cdot, x_{-\nu}^{*}\right), X_{\nu}^{\beta_{\nu}}\right)$ or, equivalently, $x_{\nu}^{*}$ is an optimal solution of the convex programming problem:

$$
\begin{array}{ll}
\operatorname{minimize}_{x_{\nu}} & \theta_{\nu}\left(x_{\nu}, x_{-\nu}^{*}\right) \\
\text { subject to } & x_{\nu} \in X_{\nu}, \quad g_{\nu}\left(x_{\nu}\right) \leq \beta_{\nu} .
\end{array}
$$

By (16) and Assumption B, for each $i$, we have either

$$
g_{\nu, i}\left(x_{\nu}^{*}\right)=\beta_{\nu, i}=-\sum_{\nu^{\prime} \neq \nu} \beta_{\nu^{\prime}, i}=-\sum_{\nu^{\prime} \neq \nu} g_{\nu^{\prime}, i}\left(x_{\nu^{\prime}}^{*}\right)
$$

implying $g_{i}\left(x_{\nu}^{*}, x_{-\nu}^{*}\right)=0$, or

$$
g_{\nu, i}\left(x_{\nu}^{*}\right)<\beta_{\nu, i}=-\sum_{\nu^{\prime} \neq \nu} \beta_{\nu^{\prime}, i}<-\sum_{\nu^{\prime} \neq \nu} g_{\nu^{\prime}, i}\left(x_{\nu^{\prime}}^{*}\right)
$$

implying $g_{i}\left(x_{\nu}^{*}, x_{-\nu}^{*}\right)<0$. Thus $x_{\nu}^{*}$ is feasible for

$$
\begin{array}{ll}
\operatorname{minimize}_{x_{\nu}} & \theta_{\nu}\left(x_{\nu}, x_{-\nu}^{*}\right) \\
\text { subject to } & x_{\nu} \in X_{\nu}, \quad g\left(x_{\nu}, x_{-\nu}^{*}\right) \leq 0,
\end{array}
$$

and the active inequality constraints at $x_{\nu}^{*}$ in (17) coincide with those in (18). Since $x_{\nu}^{*}$ is an optimal solution of (17), this implies $x_{\nu}^{*}$ is a local optimal solution of (18). Since (18) is a convex programming problem, $x_{\nu}^{*}$ is an optimal solution of (18). In view of (3), (18) is exactly $P_{\nu}\left(x_{-\nu}^{*}\right)$. This shows that (2) holds and hence $x^{*}$ is a GNE. 
Notice that, for any GNE $x^{*}$, the $\beta$ given in Theorem 3.5 satisfies the sufficient condition (16). Thus, we can refine Corollary 3.3 to

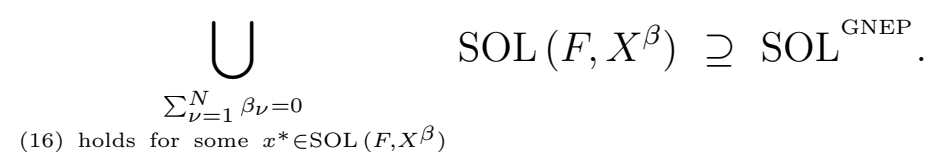

If $g_{i}\left(x^{*}\right)=0$, then a necessary condition for $x^{*} \in \operatorname{SOL}\left(F, X^{\beta}\right)$ is that $\beta_{\nu, i}=g_{\nu, i}\left(x_{\nu}^{*}\right)$ for all $\nu$. Thus, if $g_{i}\left(x^{*}\right)=0$ for many $i$ (e.g., $x^{*}$ lies in a low-dimensional face of $X$ ), then fine sampling of $\beta$ may be needed for a solution of $\operatorname{SOL}\left(F, X^{\beta}\right)$ to come near $x^{*}$.

If there exist $\underline{\beta}_{\nu} \in \Re^{m}, \nu=1, \ldots, N$, such that

$$
g_{\nu}\left(x_{\nu}\right) \geq \underline{\beta}_{\nu}, \quad \forall x_{\nu} \in X_{\nu}, \nu=1, \ldots, N,
$$

then we can further restrict $\beta$ to the bounded set

$$
\mathcal{B}:=\left\{\beta \in \Re^{N m} \mid \sum_{\nu=1}^{N} \beta_{\nu}=0, \beta_{\nu} \geq \underline{\beta}_{\nu}, \nu=1, \ldots, N\right\} .
$$

In Example 2, if we choose $g_{1}\left(x_{1}\right)=x_{1}^{2}$ and $g_{2}\left(x_{2}\right)=x_{2}^{2}-1$, then we can take as lower bounds $\underline{\beta}_{1}=0$ and $\underline{\beta}_{2}=-1$.

If a solution $x^{*}$ of $\mathrm{VI}\left(F, X^{\beta}\right)$ has a Lagrange multiplier $\lambda_{\nu}^{*}$ associated with each constraint $g_{\nu}\left(x_{\nu}\right) \leq \beta_{\nu}$, i.e., $0 \leq \lambda_{\nu}^{*} \perp g_{\nu}\left(x_{\nu}^{*}\right)-\beta_{\nu} \leq 0$, then letting

$$
\pi^{*}:=\min _{\nu=1, \ldots, N} \lambda_{\nu}^{*}, \quad \omega_{\nu}:=\lambda_{\nu}^{*}-\pi^{*}, \nu=1, \ldots, N,
$$

where the "min" is taken componentwise, we see that $\left(x^{*}, \pi^{*}\right)$ satisfies the KKT condition (11) for VI $\left(F^{\omega}, X\right)$. Thus, VI $\left(F^{\omega}, X\right)$ may be viewed as the dual of $\mathrm{VI}\left(F, X^{\beta}\right)$, with the former requiring a CQ to ensure existence of Lagrange multipliers and the latter requiring separability of shared constraints.

\subsection{Relating Price-Directed Parametrized VI to Normalized Equilibrium}

In this subsection, we relate the solutions of the price-directed parametrized VI to the following notion of a normalized equilibrium introduced by Rosen [29].

Definition 3.2. A GNE $x^{*}$ is called a normalized equilibrium if there exist $\lambda^{*} \in \Lambda\left(x^{*}\right)$ and $r=\left(r_{\nu}\right)_{\nu=1}^{N} \in \Re_{++}^{N}$ satisfying

$$
r_{1} \lambda_{1}^{*}=r_{2} \lambda_{2}^{*}=\cdots=r_{N} \lambda_{N}^{*}
$$

We say $x^{*}$ is associated with $r$. The set of normalized equilibria is denoted by $\mathrm{SOL}^{\mathrm{nE}}$.

In [29, Theorem 3], Rosen proved that there exists a normalized equilibrium associated with every $r \in \Re_{++}^{N}$, provided that $X$ is nonempty, compact, and satisfies a Slater condition. Rosen's proof is based on showing that there exists an $x^{*} \in X$ satisfying

$$
\rho_{r}\left(x^{*}, x^{*}\right)=\min _{y \in X} \rho_{r}\left(x^{*}, y\right),
$$


where $\rho_{r}: \Re^{n} \times \Re^{n} \rightarrow \Re$ is the weighted Nikaido-Isoda-type function defined by

$$
\rho_{r}(x, y):=\sum_{\nu=1}^{N} r_{\nu} \theta_{\nu}\left(y_{\nu}, x_{-\nu}\right)
$$

Notice that (20) is equivalent to $x^{*} \in \operatorname{SOL}\left(F_{r}, X\right)$, where $F_{r}: \Re^{n} \rightarrow \Re^{n}$ is defined by

$$
F_{r}(x):=\left(r_{\nu} \nabla_{x_{\nu}} \theta_{\nu}(x)\right)_{\nu=1}^{N}
$$

The Slater condition ensures that any $x^{*} \in \mathrm{SOL}\left(F_{r}, X\right)$ satisfies the KKT condition for $\min _{x \in X}\left\langle F_{r}\left(x^{*}\right), x\right\rangle$. We formally state below the relationship between normalized equilibria and the solutions of $\mathrm{VI}\left(F_{r}, X\right)$.

Proposition 3.2. For any $r \in \Re_{++}^{N}$, $x^{*}$ is a normalized equilibrium associated with $r$ if and only if $x^{*} \in \mathrm{SOL}\left(F_{r}, X\right)$ and $x^{*}$ satisfies the KKT condition for $\min _{x \in X}\left\langle F_{r}\left(x^{*}\right), x\right\rangle$.

We note that monotonicity of $F$ does not imply monotonicity of $F_{r}$, which poses challenges in solving $\mathrm{VI}\left(F_{r}, X\right)$. For example, let a linear mapping $F: \Re^{2} \rightarrow \Re^{2}$ be given by

$$
F\left(x_{1}, x_{2}\right)=\left(\begin{array}{ll}
a & b \\
c & d
\end{array}\right)\left(\begin{array}{l}
x_{1} \\
x_{2}
\end{array}\right)
$$

with $b \neq 0$. Then the mapping $F_{r}: \Re^{2} \rightarrow \Re^{2}$ with $r=(\alpha, 1)$ is not monotone for all $\alpha$ sufficiently large. This is because the symmetric part of $\left(\begin{array}{cc}\alpha a & \alpha b \\ c & d\end{array}\right)$ has determinant $\alpha a d-(\alpha b+c)^{2} / 4$ which is negative for all $\alpha$ sufficiently large.

Assuming $\Lambda\left(x^{*}\right) \neq \emptyset$ for all GNE $x^{*}$ and every optimal solution of $\min _{x \in X}\langle c, x\rangle$ satisfies its KKT condition for all $c \in \Re^{n}$, the relationships among GNEs, normalized equilibria, and the solutions of $\mathrm{VI}(F, X), \mathrm{VI}\left(F^{\omega}, X\right)$ and $\mathrm{VI}\left(F_{r}, X\right)$ can be summarized as follows:

$$
\operatorname{SOL}(F, X) \subseteq \bigcup_{r \in \Re_{++}^{N}} \operatorname{SOL}\left(F_{r}, X\right)=\operatorname{SOL}^{n E} \subseteq \mathrm{SOL}^{\mathrm{GNEP}} \subseteq \bigcup_{\omega \in \Re_{+}^{N m}} \operatorname{SOL}\left(F^{\omega}, X\right)
$$

where the first inclusion follows from the definition of $F_{r}$, the equality follows from Proposition 3.2, the second inclusion is obvious from the definition of normalized equilibria (Definition 3.2), and the last inclusion is shown in Corollary 3.1.

When the GNEP has only one shared constraint, it readily follows from Definition 3.2 that any GNE satisfying strict complementarity is a normalized equilibrium. We state this formally below.

Proposition 3.3. Suppose that $m=1$. If $x^{*}$ is a GNE satisfying strict complementarity with some $\lambda^{*} \in \Lambda\left(x^{*}\right)$ (i.e., $g_{1}\left(x^{*}\right)=0$ implies $\lambda_{\nu, 1}^{*}>0$ for all $\left.\nu=1, \ldots, N\right)$, then $x^{*}$ is a normalized equilibrium.

Thus, in the case of a single shared constraint, we can expect to find nearly all GNEs by solving $\mathrm{VI}\left(F_{r}, X\right)$ parametrized by the weights $r$. Still, we may not find all GNEs this way. In particular, in Example $1, x^{*}=(0,1)^{T}$ is a GNE with $\Lambda\left(x^{*}\right)=\left\{(2,0)^{T}\right\}$, so $x^{*}$ is not a normalized equilibrium and we will not find $x^{*}$ by solving $\mathrm{VI}\left(F_{r}, X\right)$ for 
any $r \in \Re_{++}^{N}$. In contrast, both price-directed and resource-directed parametrized VIs can find this GNE for suitable $\omega$ and $\beta$; see Corollaries 3.2 and 3.3.

In the above example with a single shared constraint, strict complementarity is violated. We can also construct examples with multiple shared constraints that satisfy strict complementarity. In particular, consider a 2-player linear-quadratic GNEP of the form

$$
\begin{array}{rll}
P_{\nu}\left(x_{-\nu}\right): & \text { minimize }_{x_{\nu}} & \frac{1}{2} x_{\nu}^{T} Q_{\nu \nu} x_{\nu}+\left(Q_{\nu,-\nu} x_{-\nu}+q_{\nu}\right)^{T} x_{\nu} \\
& \text { subject to } \quad x_{\nu} \geq 0, \quad B_{1} x_{1}+B_{2} x_{2} \leq b,
\end{array} \quad \nu=1,2,
$$

where $Q_{\nu \nu} \in \Re^{n_{\nu} \times n_{\nu}}$ is symmetric positive definite, $Q_{\nu,-\nu} \in \Re^{n_{\nu} \times n_{-\nu}}, q_{\nu} \in \Re^{n_{\nu}}, B_{\nu} \in$ $\Re^{m \times n_{\nu}}, b \in \Re^{m}$, and $m \geq 2$. Then a sufficient condition for $x^{*}=\left(x_{1}^{*}, x_{2}^{*}\right) \in \Re_{++}^{n_{1}+n_{2}}$ to be a GNE is that it, together with some $\lambda^{*}=\left(\lambda_{1}^{*}, \lambda_{2}^{*}\right) \in \Re_{+}^{2 m}$, satisfies the following linear equations (see (7)):

$$
\left(\begin{array}{cccc}
Q_{11} & Q_{12} & B_{1}^{T} & 0 \\
Q_{21} & Q_{22} & 0 & B_{2}^{T} \\
B_{1} & B_{2} & 0 & 0
\end{array}\right)\left(\begin{array}{c}
x_{1} \\
x_{2} \\
\lambda_{1} \\
\lambda_{2}
\end{array}\right)+\left(\begin{array}{c}
q_{1} \\
q_{2} \\
-b
\end{array}\right)=0
$$

Moreover, when $B_{1}$ and $B_{2}$ have rank $m, \lambda^{*}$ is uniquely determined by $x^{*}$ and $\Lambda\left(x^{*}\right)=$ $\left\{\lambda^{*}\right\}$ (since $x^{*}>0$ ). We thus have the following result.

Proposition 3.4. Consider the GNEP (21) with $m \geq 2$. Suppose that $B_{1}$ and $B_{2}$ have rank $m, x^{*}=\left(x_{1}^{*}, x_{2}^{*}\right) \in \Re_{++}^{n_{1}+n_{2}}$ together with $\lambda_{1}^{*}, \lambda_{2}^{*} \in \Re_{+}^{m}$ satisfies $(22)$, and $\lambda_{1}^{*}$ and $\lambda_{2}^{*}$ are not positive scalar multiples of each other. Then $x^{*}$ is a GNE of (21) but is not a normalized equilibrium.

Proposition 3.4 also extends to the case where only one of $B_{1}$ and $B_{2}$ has rank $m$, say $B_{2}$, and $B_{1}$ has rank of at least 1 . In this case, $\lambda_{2}^{*}$ need not be a positive scalar multiple of any $\lambda_{1} \in \Re_{+}^{m}$ satisfying $B_{1}^{T} \lambda_{1}=B_{1}^{T} \lambda_{1}^{*}$. An example is

$$
B_{1}=\left(\begin{array}{c}
-1 \\
1
\end{array}\right), \quad B_{2}=\left(\begin{array}{cc}
1 & 0 \\
0 & 1
\end{array}\right), \quad \lambda_{1}^{*}=\left(\begin{array}{l}
0 \\
1
\end{array}\right), \quad \lambda_{2}^{*}=\left(\begin{array}{l}
1 \\
0
\end{array}\right) .
$$

We then choose $x^{*}$ and $Q_{11}, Q_{12}, Q_{21}, Q_{22}$ and set $q_{1}, q_{2}, b$ according to (22). An example (corresponding to $\left.x^{*}=(1,1,1)^{T}\right)$ is

$$
\left(\begin{array}{ll}
Q_{11} & Q_{12} \\
Q_{21} & Q_{22}
\end{array}\right)=\left(\begin{array}{lll}
2 & 1 & 1 \\
1 & 2 & 0 \\
1 & 0 & 1
\end{array}\right), \quad\left(\begin{array}{l}
q_{1} \\
q_{2}
\end{array}\right)=\left(\begin{array}{l}
-5 \\
-4 \\
-2
\end{array}\right), \quad b=\left(\begin{array}{l}
0 \\
2
\end{array}\right) .
$$

It can be verified that $\mathrm{SOL}^{\mathrm{GNEP}}$ comprises the vectors

$$
\left(\begin{array}{l}
2 \\
1 \\
0
\end{array}\right), \quad\left(\begin{array}{l}
1.6 \\
1.2 \\
0.4
\end{array}\right), \quad\left(\begin{array}{c}
t \\
t \\
2-t
\end{array}\right), \quad 0 \leq t \leq \frac{4}{3}
$$


Hence the shared constraints are both active at each GNE $(t, t, 2-t)^{T}$. The $\operatorname{VI}(F, X)$ has a single solution $(2,1,0)^{T}$. The Lagrange multipliers $\lambda=\left(\lambda_{1}, \lambda_{2}\right) \in \Re^{4}$ corresponding to the above GNEs are

$$
\left(\begin{array}{l}
0 \\
0 \\
0 \\
0
\end{array}\right), \quad\left(\begin{array}{c}
0 \\
0.2 \\
0 \\
0
\end{array}\right), \quad\left(\begin{array}{c}
s \\
3-2 t+s \\
4-3 t \\
0
\end{array}\right), \quad s \geq 0,0 \leq t \leq \frac{4}{3} .
$$

Thus, among the GNEs, only $(2,1,0)^{T}$ is a normalized equilibrium. This shows that, when there are more than one active shared constraints, many of the GNEs may not be obtainable by solving $\mathrm{VI}\left(F_{r}, X\right)$ parametrized by weights $r$.

\subsection{Sampling Strategies in Parameter Spaces}

In the previous subsections we saw that we can find all GNEs by solving an (uncountably) infinite number of VIs. In practice, we can afford to solve only a finite number of VIs, with the number depending on the cost of solving each VI. Thus, we need efficient strategies for representative sampling in the space of dual parameters $\omega$ or the space of primal parameters $\beta$.

\subsubsection{Random/Grid Sampling}

One simple strategy would be to sample $\omega$ randomly or on a grid from a compact set $\Omega \subseteq \mathcal{W}$, with $\mathcal{W}$ defined by (13); see Theorem 3.4. Similar sampling strategies can be applied to sampling $\beta$ from $\mathcal{B}$ defined by (19).

\subsubsection{Adaptive Sampling}

We can improve sampling efficiency by adaptively refining the sample size on $\Omega$ or $\mathcal{B}$ or both. In what follows, we focus on $\Omega$. Using (13) and (14), $\Omega$ may be expressed as the union of the boxes

$$
\Omega_{K, \sigma}^{\rho}:=\left\{\omega=\left(\omega_{\nu}\right)_{\nu=1}^{N} \mid\left\{\begin{aligned}
\omega_{\nu, i}=0 & \text { if } i \notin K \text { or } \nu=\sigma(i) \\
\rho \geq \omega_{\nu, i} \geq 0 & \text { else }
\end{aligned}\right\} \begin{array}{l}
\nu=1, \ldots, N \\
i=1, \ldots, m
\end{array}\right\}
$$

over all $K \subseteq\{1, \ldots, m\}$ and $\sigma: K \rightarrow\{1, \ldots, N\}$, where $\rho>0$. The number of such boxes is $\sum_{|K|=0}^{m}\left(\begin{array}{c}m \\ |K|\end{array}\right) N^{|K|}=(N+1)^{m}$. In fact, the pair $(K, \sigma)$ can be more compactly represented by $\sigma:\{1, \ldots, m\} \rightarrow\{0,1, \ldots, N\}$ with $\sigma(i)=0$ indicating $i \notin K$. We first sample coarsely from each box $\Omega_{K, \sigma}^{\rho}$ (randomly or on a grid). For each $\omega$ sampled, we find an $x_{\omega}^{*} \in \operatorname{SOL}\left(F^{\omega}, X\right)$ and compute $\max _{\nu}\left|\left\langle g\left(x_{\omega}^{*}\right), \omega_{\nu}\right\rangle\right|$ as a measure of violation of the sufficient condition (14). We then sample more finely on those $\Omega_{K, \sigma}^{\rho}$ that have lesser violations (measured by the number of samples $\omega \in \Omega_{K, \sigma}^{\rho}$ whose violation is within some tolerance of the minimum violation, say) and less finely on the remaining $\Omega_{K, \sigma}^{\rho}$, and iterate. We can further partition each box into sub-boxes based on the distribution of violations at sample points, and sample more finely inside sub-boxes with lower violation. This requires more book keeping, however. 


\subsubsection{Linear-Quadratic GNEP}

When each $\theta_{\nu}$ is quadratic, $g$ is affine, and each $X_{\nu}$ is a polyhedral set, the GNEP is called linear-quadratic. Example 1 and Examples 3, 4, 5 in Section 4 are linearquadratic. In the linear-quadratic case, the set of GNEs SOL ${ }^{\text {GNEP }}$ is the union of a finite collection of polyhedral sets. Moreover, there is a close connection between each GNE and the corresponding $\omega$, which may be exploited to improve sampling efficiency, as we discuss below. Specifically, let $\theta_{\nu}, g$ and $X_{\nu}$ be given by

$$
\begin{aligned}
\theta_{\nu}(x) & =\frac{1}{2} x_{\nu}^{T} Q_{\nu, \nu} x_{\nu}+\left(Q_{\nu,-\nu} x_{-\nu}+q_{\nu}\right)^{T} x_{\nu} \\
g(x) & =\sum_{\nu=1}^{N} B_{\nu} x_{\nu}-b \\
X_{\nu} & =\left\{0 \leq x_{\nu} \leq u_{\nu} \mid A_{\nu} x_{\nu} \leq a_{\nu}\right\}
\end{aligned}
$$

where $Q_{\nu, \nu} \in \Re^{n_{\nu} \times n_{\nu}}, Q_{\nu,-\nu} \in \Re^{n_{\nu} \times n_{-\nu}}, q_{\nu} \in \Re^{n_{\nu}}, B_{\nu} \in \Re^{m \times n_{\nu}}, b \in \Re^{m}, A_{\nu} \in \Re^{l_{\nu} \times n_{\nu}}$, $a_{\nu} \in \Re^{l_{\nu}}, u_{\nu} \in(0, \infty]^{n_{\nu}}$. In particular, we assume that $Q_{\nu, \nu}$ are symmetric positive semidefinite. Then, the KKT condition (7) reduces to

$$
\begin{array}{ll}
0=\operatorname{mid}\left\{x_{\nu}, \Theta_{\nu}\left(x, \lambda_{\nu}, \mu_{\nu}\right), x_{\nu}-u_{\nu}\right\}, & \nu=1, \ldots, N \\
0 \leq \mu_{\nu} \perp A_{\nu} x_{\nu}-a_{\nu} \leq 0, & \nu=1, \ldots, N \\
0 \leq \lambda_{\nu} \perp \sum_{\nu^{\prime}=1}^{N} B_{\nu^{\prime}} x_{\nu^{\prime}}-b \leq 0, & \nu=1, \ldots, N
\end{array}
$$

where we denote for simplicity $\Theta_{\nu}\left(x, \lambda_{\nu}, \mu_{\nu}\right)=Q_{\nu} x+q_{\nu}+A_{\nu}^{T} \mu_{\nu}+B_{\nu}^{T} \lambda_{\nu}$ with $Q_{\nu}=$ $\left(Q_{\nu, \nu}, Q_{\nu,-\nu}\right)$, and mid means taking the median componentwise. Then the solution set of the above system can be expressed as the disjoint union of the polyhedral sets

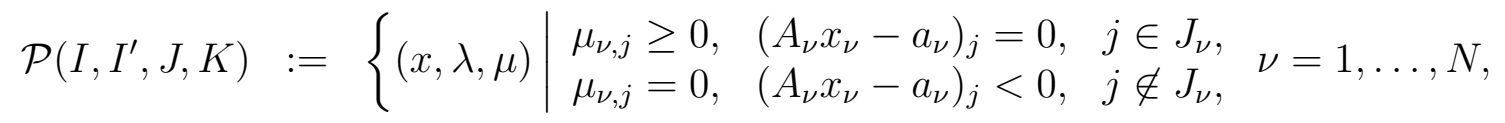

$$
\begin{aligned}
& \lambda_{\nu, k} \geq 0, \quad\left(\sum_{\nu^{\prime}=1}^{N} B_{\nu^{\prime}} x_{\nu^{\prime}}-b\right)_{k}=0, \quad k \in K, \\
& \lambda_{\nu, k}=0, \quad\left(\sum_{\nu^{\prime}=1}^{N} B_{\nu^{\prime}} x_{\nu^{\prime}}-b\right)_{k}^{k}<0, \quad k \notin K, \quad \nu=1, \ldots, N, \\
& x_{\nu, i}=0, \quad \Theta_{\nu}\left(x, \lambda_{\nu}, \mu_{\nu}\right)_{i} \geq 0, \quad i \in I_{\nu}, \\
& x_{\nu, i}=u_{\nu, i}, \quad \Theta_{\nu}\left(x, \lambda_{\nu}, \mu_{\nu}\right)_{i} \leq 0, \quad i \in I_{\nu}^{\prime} \text {, } \\
& 0<x_{\nu, i}<u_{\nu, i}, \quad \Theta_{\nu}\left(x, \lambda_{\nu}, \mu_{\nu}\right)_{i}=0, \quad i \notin I_{\nu} \cup I_{\nu}^{\prime}, \\
& \nu=1, \ldots, N\}
\end{aligned}
$$

over all $I=\left\{I_{\nu}\right\}_{\nu=1}^{N}, I^{\prime}=\left\{I_{\nu}^{\prime}\right\}_{\nu=1}^{N}, J=\left\{J_{\nu}\right\}_{\nu=1}^{N}$, and $K \subseteq\{1, \ldots, m\}$, with $I_{\nu}, I_{\nu}^{\prime} \subseteq$ $\left\{1, \ldots, n_{\nu}\right\}$ such that $I_{\nu} \cap I_{\nu}^{\prime}=\emptyset$ and $J_{\nu} \subseteq\left\{1, \ldots, l_{\nu}\right\}, \nu=1, \ldots, N$. If $Q=\left(Q_{\nu}\right)_{\nu=1}^{N^{\nu}}$ is positive definite, as in Examples 1, 3 and 4, then $F$ defined by (4) is strongly monotone, and so, by Proposition 3.1, SOL $\left(F^{\omega}, X\right)$ is a singleton for any $\omega \in \mathcal{W}$.

If $\mathcal{P}\left(I, I^{\prime}, J, K\right)$ is nonempty, then its projection onto the $x$-space lies in the relative interior of one of the faces of $X$, and this face corresponds uniquely to $\left(I, I^{\prime}, J, K\right)$. By (11) and Theorem 3.3, for any $\omega \in \mathcal{W}$ and $x \in \mathrm{SOL}\left(F^{\omega}, X\right)$ with associated multipliers $\pi$ and $\mu=\left(\mu_{\nu}\right)_{\nu=1}^{N}, x$ is a GNE if and only if $\left(x,\left(\omega_{\nu}+\pi\right)_{\nu=1}^{N}, \mu\right) \in \mathcal{P}\left(I, I^{\prime}, J, K\right)$ for some $I, I^{\prime}, J, K$. Since each $\Omega_{K, \sigma}^{\rho}$ is convex, this implies that, for any $\omega, \omega^{\prime} \in \Omega_{K, \sigma}^{\rho}$, if $x \in \operatorname{SOL}\left(F^{\omega}, X\right)$ and $x^{\prime} \in \operatorname{SOL}\left(F^{\omega^{\prime}}, X\right)$ are both GNEs that lie in the relative interior of the same face of $X$, then $\alpha x+(1-\alpha) x^{\prime}(0 \leq \alpha \leq 1)$ is also a GNE lying in the the same 
face and it belongs to SOL $\left(F^{\alpha \omega+(1-\alpha) \omega^{\prime}}, X\right)$. Thus, for any $K, \sigma$ and any $\omega^{1}, \ldots, \omega^{t} \in$ $\Omega_{K, \sigma}^{\rho}$, if the corresponding parameterized VI solutions $x^{1}, \ldots, x^{t}$ are GNE and lie in the relative interior of the same face of $X$, then any convex combination of $x^{1}, \ldots, x^{t}$ is also a GNE lying in the same face of $X$ and it solves a parameterized VI corresponding to the same convex combination of $\omega^{1}, \ldots, \omega^{t}$. Hence we can save computation by not sampling inside the convex hull of $\omega^{1}, \ldots, \omega^{t}$. Checking membership in the convex hull is relatively easy when $\Omega_{K, \sigma}^{\rho}$ has dimension 1 or 2 as in Examples 1, 3 and 4 . In a higher dimensional case, linear programming may be used. When $m$ and $n$ are small, we can determine which $\mathcal{P}\left(I, I^{\prime}, J, K\right)$ is nonempty using linear programming and then find all vertices of $\mathcal{P}\left(I, I^{\prime}, J, K\right)$ using a vertex enumeration algorithm; see [3] and references therein.

\subsubsection{Sequential Linear-Quadratic Approximations}

If the GNEP is not linear-quadratic, we can locally approximate each $\theta_{\nu}$ by a convex quadratic function $\tilde{\theta}_{\nu}$, approximate $g$ by an affine function $\tilde{g}$, approximate each $X_{\nu}$ by a polyhedral set $\tilde{X}_{\nu}$, and find some GNE $x^{*}$ (or, if practical, find all GNEs) for this approximate problem with associated Lagrange multipliers $\lambda^{*}=\left(\lambda_{\nu}^{*}\right)_{\nu=1}^{N}$. If $\nabla \theta_{\nu}\left(x_{\nu}^{*}\right) \approx$ $\nabla \tilde{\theta}_{\nu}\left(x_{\nu}^{*}\right)$ for all $\nu$ and $\nabla g\left(x^{*}\right) \approx \nabla \tilde{g}\left(x^{*}\right)$, then use $\omega$ given by (12) as parameter for $\mathrm{VI}\left(F^{\omega}, X\right)$. Otherwise, refine the local approximation around $x^{*}$, and repeat. This approach is in the spirit of the Josephy-Newton method for solving VI [18]. In fact, it may be possible to improve the efficiency of sampling by combining this approach with implicit function theorem for parameterized VI; see [5, 9, 27] and references therein. This is a direction for future study.

\section{Implementation and Numerical Results}

In this section, we report our implementation of and numerical experience with the parametrized VI approaches. For our tests, we use three linear-quadratic GNEPs taken from the literature, which are described below. Our implementation and numerical results are reported in Subsections 4.2 and 4.3.

\subsection{Test Examples}

Example 3 (Harker's example). This problem is taken from [13]. There are two players and they solve the following problems:

$$
\begin{aligned}
& P_{1}\left(x_{2}\right): \quad \operatorname{minimize}_{x_{1}} \quad x_{1}^{2}+\frac{8}{3} x_{1} x_{2}-34 x_{1} \quad P_{2}\left(x_{1}\right): \quad \operatorname{minimize}_{x_{2}} \quad x_{2}^{2}+\frac{5}{4} x_{1} x_{2}-24.25 x_{2} \\
& \text { subject to } 0 \leq x_{1} \leq 10 \quad \text { subject to } 0 \leq x_{2} \leq 10 \\
& x_{1}+x_{2} \leq 15, \quad x_{1}+x_{2} \leq 15 .
\end{aligned}
$$

This is a GNEP with one shared constraint and the solution set is given by

$$
\mathrm{SOL}^{\mathrm{GNEP}}=\left\{\left(\begin{array}{l}
5 \\
9
\end{array}\right)\right\} \cup\left\{\left(\begin{array}{c}
t \\
15-t
\end{array}\right) \mid 9 \leq t \leq 10\right\} .
$$


The corresponding $F: \Re^{2} \rightarrow \Re^{2}$ and $X \subseteq \Re^{2}$ are represented as

$$
\begin{aligned}
F(x) & =\left(\begin{array}{c}
2 x_{1}+\frac{8}{3} x_{2}-34 \\
\frac{5}{4} x_{1}+2 x_{2}-24.25
\end{array}\right), \\
X & =\left\{x \in \Re^{2} \mid x_{1}+x_{2} \leq 15,0 \leq x_{\nu} \leq 10, \nu=1,2\right\} .
\end{aligned}
$$

Since $F$ is strongly monotone on $\Re^{2}$, VI $(F, X)$ has a unique solution, which is given by $x=(5,9)^{T}$. Note that this solution lies in the interior of the set $X$.

Example 4 (River basin pollution game). Consider the 3-person river basin pollution game studied in [19, Section 5.3], where the problem of player $\nu \in\{1,2,3\}$ is defined by

$$
\begin{aligned}
P_{\nu}\left(x_{-\nu}\right): \text { minimize }_{x_{\nu}} & \left(\alpha_{\nu} x_{\nu}+0.01\left(x_{1}+x_{2}+x_{3}\right)-\chi_{\nu}\right) x_{\nu} \\
\text { subject to } & x_{\nu} \geq 0 \\
& 3.25 x_{1}+1.25 x_{2}+4.125 x_{3} \leq 100 \\
& 2.2915 x_{1}+1.5625 x_{2}+2.8125 x_{3} \leq 100
\end{aligned}
$$

with $\alpha_{1}=0.01, \alpha_{2}=0.05, \alpha_{3}=0.01, \chi_{1}=2.9, \chi_{2}=2.88, \chi_{3}=2.85$. This GNEP has two shared constraints. The corresponding $F: \Re^{3} \rightarrow \Re^{3}$ and $X \subseteq \Re^{3}$ are given by

$$
\begin{aligned}
F(x) & =\left(\begin{array}{lll}
0.04 & 0.01 & 0.01 \\
0.01 & 0.12 & 0.01 \\
0.01 & 0.01 & 0.04
\end{array}\right) x-\left(\begin{array}{c}
2.9 \\
2.88 \\
2.85
\end{array}\right), \\
X & =\left\{x \in \Re_{+}^{3} \mid\left(\begin{array}{ccc}
3.25 & 1.25 & 4.125 \\
2.2915 & 1.5625 & 2.8125
\end{array}\right) x \leq\left(\begin{array}{c}
100 \\
100
\end{array}\right)\right\} .
\end{aligned}
$$

Since $F$ is strongly monotone on $\Re^{3}$, VI $(F, X)$ has a unique solution, which is given by $x=\left(\frac{4673}{221}, \frac{5754}{359}, \frac{567}{208}\right)^{T} \approx(21.14,16.03,2.73)^{T}$. Note that, at this solution, the first inequality defining $X$ is active, while the second inequality is inactive.

Example 5 (Electricity market model). Consider a model from [24, Section 5.3 and Erratum] of electricity markets with endogenous arbitrage. The model consists of $N(N \geq 2)$ electricity firms competing on a spatial network of markets along with an arbitrager who attempts to make a profit by exploiting price differentials between regions. In the original model [16], each firm maximizes its profit with anticipating the arbitragers' optimal response, resulting in a multi-leader-follower-game. In [24, Section 5.3], the arbitrager is removed from the model and the price differentials are assumed to be less than the shipping costs. In this setting, the model can be formulated as a GNEP as described below. We make a slight (and reasonable) modification to the model in [24, Section 5.3] by setting the shipping costs from nodes to themselves to zero. Interestingly, this also results in some significant GNEs being found; see the discussions in Subsection 4.3.

The regions are represented by the nodes in a network and each firm has electricity plants at those nodes. Each firm determines how much it should produce at each plant and how much it should sell at each node to maximize its profit. We introduce the notations to formulate the problem.

\section{Problem Data}


Table 1: Generation costs $c_{\nu, i}$ and capacities $\mathrm{CAP}_{\nu, i}$

\begin{tabular}{ccccccc}
\hline$(\nu, i)$ & $(1,1)$ & $(1,2)$ & $(1,3)$ & $(2,1)$ & $(2,2)$ & $(2,3)$ \\
\hline$c_{\nu, i}$ & 15 & 15 & 15 & 15 & 15 & 15 \\
$\mathrm{CAP}_{\nu, i}$ & 100 & 50 & 0 & 0 & 100 & 50 \\
\hline
\end{tabular}

$\begin{array}{lll}\mathcal{N} & : & \text { set of nodes } \\ \mathcal{A} \subseteq \mathcal{N} \times \mathcal{N} & : & \text { set of arcs with price differential constraint } \\ c_{\nu, i} & : & \text { cost per unit generation at node } i \text { by firm } \nu \\ P_{i} & : & \text { price intercept of sales function at node } i \\ Q_{i} & : \text { quantity intercept of sales function at node } i \\ e_{i j} & : \text { unit cost of shipping from node } i \text { to } j \\ \operatorname{CAP}_{\nu, i} & : \text { production capacity at node } i \text { for firm } \nu\end{array}$

\section{Variables}

$x_{\nu, i j}$ : amount produced at node $i$ and sold at node $j$ by firm $\nu$

$S_{j} \quad:$ amount of total sales at node $j$

$$
S_{j}:=\sum_{\nu=1}^{N} \sum_{i \in \mathcal{N}} x_{\nu, i j}, \quad \forall j \in \mathcal{N}
$$

$p_{j} \quad: \quad$ market price at node $j$

$$
p_{j}\left(S_{j}\right):=P_{j}-\frac{P_{j}}{Q_{j}} S_{j}, \quad \forall j \in \mathcal{N}
$$

Each firm $\nu$ 's problem is to find $\left\{x_{\nu, i j}\right\}_{(i, j) \in \mathcal{A}}$ that solve the following minimization problem for a given $\left\{x_{\nu^{\prime}, i j}\right\}_{\nu^{\prime} \neq \nu,(i, j) \in \mathcal{A}}$,

$$
\begin{array}{ll}
\operatorname{minimize}_{x_{\nu, i j}} & \sum_{i \in \mathcal{N}} \sum_{j \in \mathcal{N}}\left(c_{\nu, i}-p_{j}\left(S_{j}\right)\right) x_{\nu, i j}+\sum_{(i, j) \in \mathcal{A}} e_{i j} x_{\nu, i j} \\
\text { subject to } \quad & \sum_{j \in \mathcal{N}} x_{\nu, i j} \leq \mathrm{CAP}_{\nu, i}, \quad \forall i \in \mathcal{N} \\
& p_{j}\left(S_{j}\right)-p_{i}\left(S_{i}\right) \leq e_{i j}, \quad \forall(i, j) \in \mathcal{A} \\
& x_{\nu, i j} \geq 0, \quad \forall(i, j) \in \mathcal{A} .
\end{array}
$$

This is a 2-player GNEP with $|\mathcal{A}|$ shared constraints, because the price differential constraints $p_{j}\left(S_{j}\right)-p_{i}\left(S_{i}\right) \leq e_{i j}$ depend on the total sales at each node $S_{j}=\sum_{\nu=1}^{N} \sum_{i \in \mathcal{N}} x_{\nu, i j}$.

In our test, we use the same data as in [24, Section 5.3 and Erratum], with $N=2$ firms, node set $\mathcal{N}=\{1,2,3\}$, arc set $\mathcal{A}=\{(1,2),(1,3),(2,1),(2,3),(3,1),(3,2)\}$, and $e_{i j}=1$ for all $(i, j) \in \mathcal{A}$. The remaining data are shown in Tables 1 and 2 . Here firm 1 owns electricity plants at nodes 1 and 2 , and firm 2 owns electricity plants at nodes 2 and 3. Hence $\mathrm{CAP}_{1,3}=\mathrm{CAP}_{2,1}=0$, so that $x_{1,31}=x_{1,32}=x_{1,33}=x_{2,11}=x_{2,12}=$ $x_{2,13}=0$.

\subsection{Implementation}

We now describe our implementation of the parametrized VI approaches for a linearquadratic GNEP of the form (24), (25), (26). 
Table 2: Price function data $\left(P_{i}, Q_{i}\right)$

\begin{tabular}{cccc}
\hline node $i$ & 1 & 2 & 3 \\
\hline$P_{i}$ & 40 & 35 & 32 \\
$Q_{i}$ & 500 & 400 & 600 \\
\hline
\end{tabular}

For the price-directed parametrized VI, we enumerate all $(N+1)^{m}$ mappings $\sigma:\{1, \ldots, m\} \rightarrow\{0,1, \ldots, N\}$ and, for each $\sigma$, we set $K=\{i \mid \sigma(i) \neq 0\}$. The corresponding $\Omega_{K, \sigma}^{\rho}$ given by (23) is a Cartesian product of $(N-1)|K|$ intervals of the form $[0, \rho]$ and we accordingly generate $N_{s}^{(N-1)|K|}$ samples of $\omega=\left(\omega_{\nu}\right)_{\nu=1}^{N}$ from $\Omega_{K, \sigma}^{\rho}$ ( $N_{s}$ is a user-chosen positive integer) by setting $\omega_{\nu, i}=0$ if $i \notin K$ or $\sigma(i)=\nu$ and otherwise generate $\omega_{\nu, i}$ (either on a grid or randomly according to the uniform distribution) from $(0, \rho]$. Since there are $N^{|K|}$ different mappings $\sigma: K \rightarrow\{0,1, \ldots, N\}$, the total number of samples is $\sum_{|K|=0}^{m}\left(\begin{array}{c}m \\ |K|\end{array}\right) N^{|K|} N_{s}^{(N-1)|K|}=\left(N N_{s}^{N-1}+1\right)^{m}$. When $m \geq 5$ such as in Example 5, this number is large even for $N_{s}=10$. To improve sampling efficiency, we abort sampling for a given $K$ and $\sigma$ if no GNE is found after 200 samples from $\Omega_{K, \sigma}^{\rho}$. We also restrict $|K|$ to be below 3. Upon solving $\mathrm{VI}\left(F^{\omega}, X\right)$ to obtain a solution $x^{*}$, we declare $x^{*}$ to be a GNE if it satisfies the condition (14) approximately, i.e.,

$$
\left|\left\langle\omega_{\nu}, g\left(x^{*}\right)\right\rangle\right| \leq 10^{-6}, \quad \nu=1, \ldots, N
$$

For the resource-directed parametrized VI, we exploit the structure of the shared constraints (25) and set $g_{\nu}\left(x_{\nu}\right):=B_{\nu} x_{\nu}-\frac{b}{N}$. Moreover, for $x_{\nu} \in X_{\nu}$, we have $0 \leq x_{\nu} \leq$ $u_{\nu}$ and hence

$$
g_{\nu}\left(x_{\nu}\right) \geq \underline{\beta}_{\nu}:=\min \left\{B_{\nu}, 0\right\} u_{\nu}-\frac{b}{N},
$$

where "min" is taken entrywise. We can use the above $\underline{\beta}_{\nu}$ in the sampling set $\mathcal{B}$ defined by (19). To simplify sampling, we take the minimum of $\underline{\beta}_{\nu, i}$ over all $\nu$, call it $\beta_{i}^{\min }$, for all $i=1, \ldots, m$, and use $\beta^{\min }=\left(\beta_{1}^{\min }, \cdots, \beta_{m}^{\min }\right)^{T}$ in place of $\underline{\beta}_{\nu}$ in (19). This yields a slightly larger $\mathcal{B}$, but one with a simpler structure. In particular, $\mathcal{B}$ is the Cartesian product of $m$ simplices of dimension $N-1$ each, with the $i$ th simplex being the convex hull of $(1-N, 1, \ldots, 1)^{T},(1,1-N, \ldots, 1)^{T}, \ldots,(1, \ldots, 1,1-N)^{T}$, scaled by $\beta_{i}^{\min }$. We generate $N$ nonnegative weights $w_{1}, \cdots, w_{N}$ from the unit simplex (either on a grid or randomly according to the uniform distribution), and take $\left(\beta_{1, i}, \ldots, \beta_{N, i}\right)$ to be the sum of the preceding $N$ points weighted by $w_{1}, \cdots, w_{N}$. We generate a total of $\left(\Gamma_{N_{s}}^{N}\right)^{m}$ samples of $\beta$ from $\mathcal{B}$, where $\Gamma_{N_{s}}^{N}$ denotes the number of grid points in the $N$-dimensional unit simplex with $N_{s}$ grid points per dimension. It is easily verified that $\Gamma_{N_{s}}^{2}=N_{s}$ and $\Gamma_{N_{s}}^{3}=N_{s}\left(N_{s}+1\right) / 2$. In general, $\Gamma_{N_{s}}^{N}=O\left(N_{s}^{N-1}\right)$. Analogous to price-directed parametrized VI, we can restrict our sampling space by replacing $\beta_{i}^{\text {min }}$ with $\max \left\{\beta_{i}^{\min },-\rho\right\}$, where $\rho>0$ is user-chosen.

Upon solving $\mathrm{VI}\left(F, X^{\beta}\right)$ to obtain a solution $x^{*}$, we declare $x^{*}$ to be a GNE if it satisfies the condition (16) approximately, i.e.,

$$
\text { for each } i=1, \ldots, m, \quad\left\{\begin{array}{lll}
\text { either } & \left|g_{\nu, i}\left(x_{\nu}^{*}\right)-\beta_{\nu}\right|<10^{-6}, \quad \nu=1, \ldots, N \\
\text { or } & g_{\nu, i}\left(x_{\nu}^{*}\right)-\beta_{\nu}<-10^{-6}, \quad \nu=1, \ldots, N .
\end{array}\right\}
$$


For a linear-quadratic GNEP of the form (24)-(26), both VI $\left(F^{\omega}, X\right)$ and VI $\left(F, X^{\beta}\right)$ can be converted to equivalent (mixed) linear complementarity problems (LCPs) of the form $\operatorname{mid}\{z, M z+c, z-d\}=0$, with

$M=\left(\begin{array}{ccc}Q & B^{T} & A^{T} \\ -B & 0 & 0 \\ -A & 0 & 0\end{array}\right), c=\left(\begin{array}{c}r \\ s \\ a\end{array}\right), d=\left(\begin{array}{c}u \\ \infty \\ \infty\end{array}\right), A=\left(\begin{array}{ccc}A_{1} & & \\ & \ddots & \\ & & A_{N}\end{array}\right), a=\left(\begin{array}{c}a_{1} \\ \vdots \\ a_{N}\end{array}\right)$,

and

$$
\begin{array}{llll}
\text { either } & B=\left(\begin{array}{lll}
B_{1} & \cdots & B_{N}
\end{array}\right), \quad s=b, & \\
\text { or } & B=\left(\begin{array}{ccc}
B_{1} & & \\
& \ddots & \\
& & B_{N}
\end{array}\right), \quad s=\left(\begin{array}{c}
\beta_{1}+\frac{b}{N} \\
\vdots \\
\beta_{N}+\frac{b}{N}
\end{array}\right), \quad r=q .
\end{array}
$$

In our tests, the LCP is solved by the MATLAB code PATHLCP.M [11] though other LCP solvers can also be used. For example, it may be more efficient to resolve the LCPs using warm starts. Since VI $\left(F, X^{\beta}\right)$ may be infeasible so that the LCP has no solution, the solver must be able to detect this when resource-directed parametrization is used.

\subsection{Numerical Results}

We now report our numerical experience with the parametrized VI approaches on the three test examples: Harker's example, the river basin pollution game and the electricity market model. The runs are made on an HP DL360 workstation under Matlab 7.2. Two GNEs are judged to be distinct when their 1-norm distance exceeds $10^{-5}$.

Example 3 (Harker's example, continued). For this example, $N=n=2$ and $m=1$.

For the price-directed parametrization approach, we sample $N_{s}=256$ points per interval $[0, \rho]$ with $\rho=2$. The sample space for $\omega$ comprises the origin $(0,0)^{T}$ and $\left\{\omega \in[0,2]^{2} \mid \omega_{2}=0\right\},\left\{\omega \in[0,2]^{2} \mid \omega_{1}=0\right\}$. We thus solve at most $2 \cdot 256+1=$ 513 LCPs and declare a solution $x^{*}$ to be GNE if it satisfies (4.2). When sampled on a grid, 458 LCPs are solved, with 98 of the LCPs yielding GNE, as plotted in Figure 1. The number of distinct GNEs is 13. Run time is below a second. We observe that the GNEs found are widely distributed over the set of GNEs given by (27). In contrast, the VI approach of $[6,7,32]$ can only find the GNE $x=(5,9)^{T}$, which is a variational/normalized equilibrium. When sampled randomly, the number of GNEs found tends to be higher, around 100, and the number of distinct GNEs is around 15.

For the resource-directed parametrization approach, we sample $N_{s}=256$ points per interval $[0,1]$, with $\rho=\infty$, and $g_{1}\left(x_{1}\right)=x_{1}-\frac{15}{2}$ and $g_{2}\left(x_{2}\right)=x_{2}-\frac{15}{2}$. The parametrized feasible set is

$$
X^{\beta}=\left\{\left(\begin{array}{l}
x_{1} \\
x_{2}
\end{array}\right) \mid x_{\nu}-\frac{15}{2} \leq \beta_{\nu}, 0 \leq x_{\nu} \leq 10, \nu=1,2\right\} .
$$




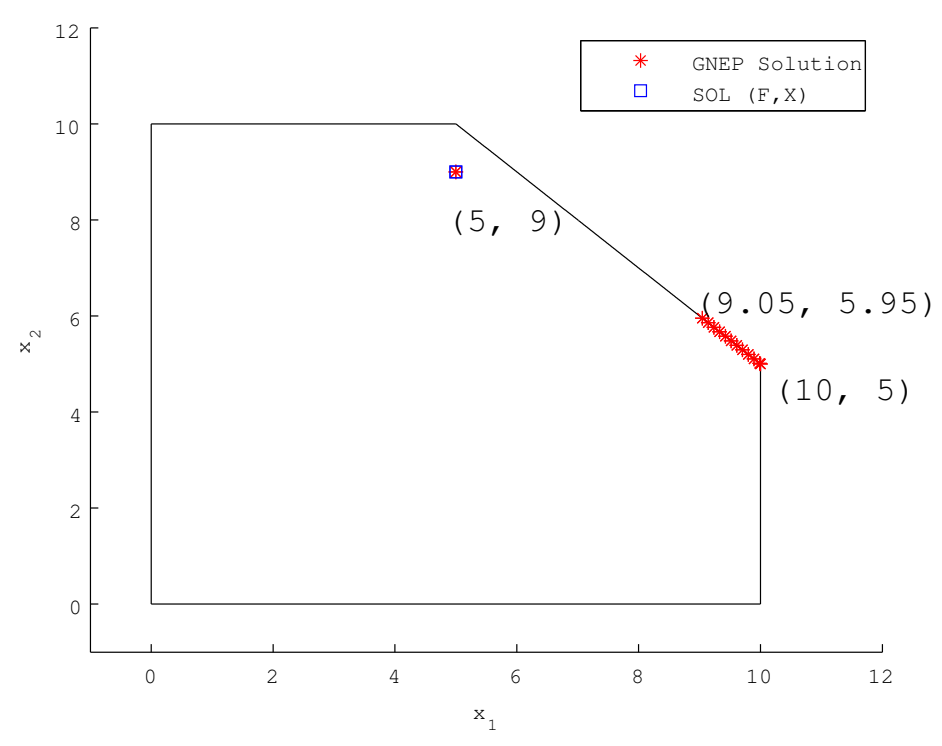

Figure 1: GNEs found by the price-directed parametrization approach for Harker's example.

Here $\beta^{\min }=-\frac{15}{2}$ and $\beta$ is sampled from the bounded set

$$
\left\{\left(\begin{array}{l}
\beta_{1} \\
\beta_{2}
\end{array}\right) \in \Re^{2} \mid \beta_{1}+\beta_{2}=0, \beta_{\nu} \geq-\frac{15}{2}, \nu=1,2\right\}=\operatorname{conv}\left\{\left(\begin{array}{c}
7.5 \\
-7.5
\end{array}\right),\left(\begin{array}{c}
-7.5 \\
7.5
\end{array}\right)\right\} .
$$

We solve 256 LCPs and declare a solution $x^{*}$ to be a GNE if it satisfies (4.2). When sampled on a grid, 34 of the LCPs yield GNEs, as plotted in Figure 2. Among these 34 GNEs, 16 equal $(5,9)^{T}$, and the remaining 18 GNEs lie on the face $x_{1}+x_{2}=15$ of $X$. The number of distinct GNEs is 19. Run time is below half a second. We observe that the GNEs found are also widely distributed over the set of GNEs given by (27), and the GNE yield rate is higher compared to price-directed parametrization (19 out of 256 versus 13 out of 458). When sampled randomly, the number of GNEs found tends to be higher, around 50, and the number of distinct GNEs is around 20.

Example 4 (River basin pollution game, continued). For this example, $N=$ $n=3$ and $m=2$.

For the price-directed parametrization approach, we sample $N_{s}=20$ points per interval $[0, \rho]$ with $\rho=2$. The sample space for $\omega$ comprises $(N+1)^{m}=16$ sets of the form (23), with $K \subseteq\{1,2\}$ and $\sigma: K \rightarrow\{1,2,3\}$. These are

(a) the origin $(0,0,0,0,0,0)^{T}$,

(b) 1,000 points from $\Omega_{\{1\}, \sigma}^{2}$ for each $\sigma:\{1\} \rightarrow\{1,2,3\}$,

(c) 1,000 points from $\Omega_{\{2\}, \sigma}^{2}$ for each $\sigma:\{2\} \rightarrow\{1,2,3\}$.

(d) 10,000 points from $\Omega_{\{1,2\}, \sigma}^{2}$ for each $\sigma:\{1,2\} \rightarrow\{1,2,3\}$. 


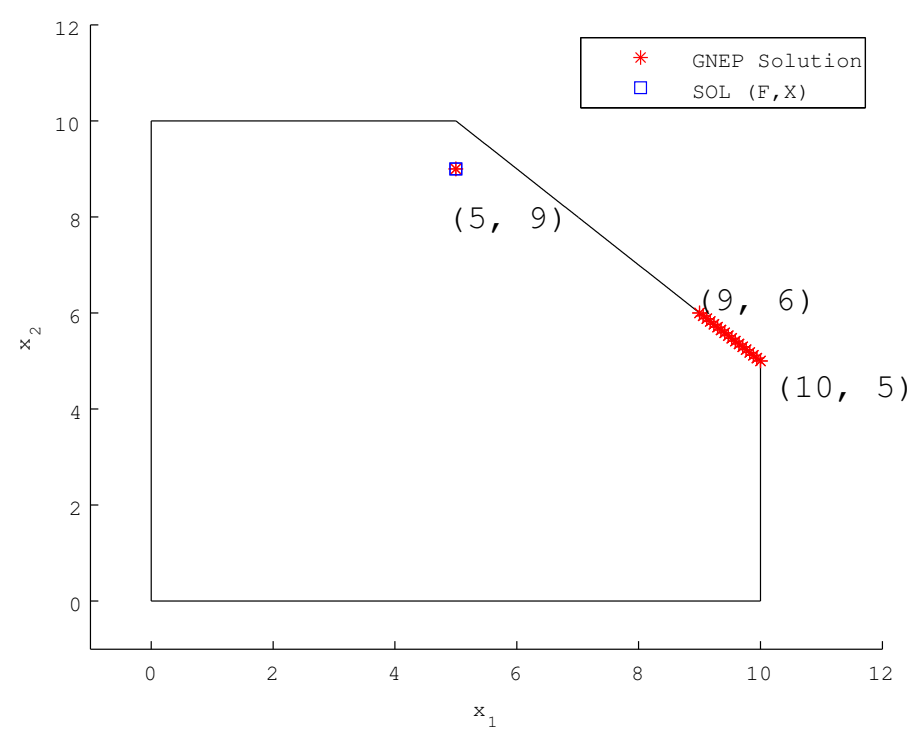

Figure 2: GNEs found by the resource-directed parametrization approach for Harker's example.

We thus solve at most $\left(3 \cdot 20^{2}+1\right)^{2}=1442401$ LCPs and declare a solution $x^{*}$ to be a GNE if it satisfies (4.2). When sampled on a grid, 3613 LCPs are solved, with 509 of the LCPs yielding GNE, as plotted in Figure 3. The number of distinct GNEs is 113. Run time is $6-7$ seconds. Note that the polyhedral set in Figure 3 represents the feasible region $X$. We obtained the variational/normalized equilibrium $x^{*}=(21.14,16.03,2.73)^{T}$ by setting $\omega=(0,0,0,0,0,0)^{T}$ and the remaining GNEs were found in case (c). Thus, the shared constraint $3.25 x_{1}+1.25 x_{2}+4.125 x_{3} \leq 100$ is active at any of these GNEs. In contrast, existing methods find only the above variational equilibrium [15, Table 3], [19, page 70], [20, page 199]. When sampled randomly, the number of GNEs found tends to be higher, around 900, and the number of distinct GNEs is around 800. Run time remains $6-7$ seconds.

For the resource-directed parametrization approach, we sample $N_{s}=20$ points per interval $[0,1]$, with $\rho=\infty$, and $g_{\nu, i}\left(x_{\nu}\right)=\gamma_{\nu, i} x_{\nu}-\frac{100}{3}$ for $\nu=1,2,3$ and $i=1,2$, where $\gamma_{1,1}=3.25, \gamma_{2,1}=1.25, \gamma_{3,1}=4.125, \gamma_{1,2}=2.2915, \gamma_{2,2}=1.5625, \gamma_{3,2}=2.8125$. The parametrized feasible set is

$$
X^{\beta}=\left\{\left(\begin{array}{l}
x_{1} \\
x_{2} \\
x_{3}
\end{array}\right) \mid \gamma_{\nu, i} x_{\nu}-\frac{100}{3} \leq \beta_{\nu, i}, x_{\nu} \geq 0, i=1,2, \nu=1,2,3\right\} .
$$

Here $\beta^{\min }=\left(-\frac{100}{3},-\frac{100}{3}\right)^{T}$ and $\beta$ is sampled from the bounded set

$$
\begin{aligned}
& \left\{\left(\beta_{\nu, i}\right) \in \Re^{6} \mid \beta_{1, i}+\beta_{2, i}+\beta_{3, i}=0, \beta_{\nu, i} \geq-\frac{100}{3}, \nu=1,2,3, i=1,2\right\} \\
= & \operatorname{conv}\left\{\left(\begin{array}{c}
200 / 3 \\
-100 / 3 \\
-100 / 3
\end{array}\right),\left(\begin{array}{c}
-100 / 3 \\
200 / 3 \\
-100 / 3
\end{array}\right),\left(\begin{array}{c}
-100 / 3 \\
-100 / 3 \\
200 / 3
\end{array}\right)\right\}^{2} .
\end{aligned}
$$



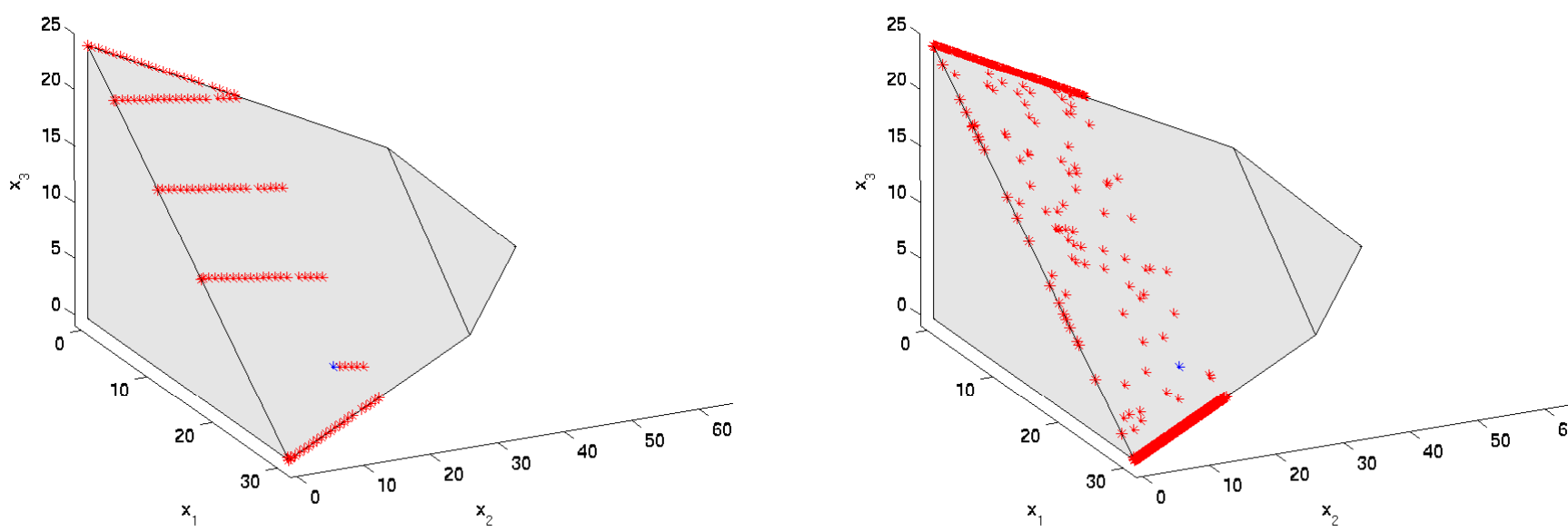

Figure 3: GNEs found by the price-directed parametrization approach, using grid sampling (left) and random sampling (right), for Example 4.

We thus solve $\left(\frac{20 \cdot 21}{2}\right)^{2}=44100$ LCPs and declare a solution $x^{*}$ to be a GNE if it satisfies (4.2). When sampled on a grid, 994 of the LCPs yield GNEs, as plotted in Figure 4. The number of distinct GNEs is 105. Run time is about 76 seconds. We observe that the GNEs found are also widely distributed over the face determined by the shared constraint $3.25 x_{1}+1.25 x_{2}+4.125 x_{3}=100$. When sampled randomly, the number of GNEs found tends to be higher, around 1400, and the number of distinct GNEs is also around 1400. Run time is around 60 seconds.

The yield rate for GNE is lower for resource-directed parametrization than for price-directed parametrization. However, upon comparing Figures 3 and 4, we see that GNEs are spatially more uniformly distributed in the latter, whereas GNEs are concentrated at the top and bottom edges in the former. This may be because pricedirected parametrization changes the normal of the supporting hyperplane to $X$ and, since $X$ is a polyhedral set, the same face is supported by many different hyperplanes. If $X$ is a strictly convex body with smooth boundary, then the GNEs found might be more spread out. In contrast, resource-directed parametrization divides up the feasible set relatively uniformly and finds GNEs within each subdivision.

Example 5 (Electricity market model, continued). For this example, $N=2$, $n_{1}=n_{2}=6$, and $m=6$.

For the price-directed parametrization approach, we sample $N_{s}=20$ points per interval $[0, \rho]$ with $\rho=20$. The sample space for $\omega$ comprises $(N+1)^{m}=3^{6}=729$ sets of the form (23), with $K \subseteq \mathcal{A}$ and $\sigma: K \rightarrow\{1,2\}$. But this is still too many, so we further restrict $K$ to those with $|K| \leq 2$. This yields the origin $(0, \ldots, 0)^{T}$ as well as the $2 \cdot 6=12$ line segments

$$
\Omega_{\{(i, j)\}, \sigma}^{20}:=\left\{\omega \in[0,20]^{12} \mid \omega_{\nu, i j} \in[0,20] \text { and } \omega_{\nu^{\prime}, k l}=0 \forall\left(\nu^{\prime}, k, l\right) \neq(\nu, i, j)\right\},
$$

where $\nu=\sigma((i, j)) \in\{1,2\}$ and $(i, j) \in \mathcal{A}$, and the $2^{2} \cdot\left(\begin{array}{l}6 \\ 2\end{array}\right)=60$ boxes

$$
\Omega_{\left\{(i, j),\left(i^{\prime}, j^{\prime}\right)\right\}, \sigma}^{20}:=\left\{\begin{array}{l|l}
\omega \in[0,20]^{12} & \begin{array}{l}
\omega_{\nu, i j} \in[0,20], \omega_{\nu^{\prime}, i^{\prime} j^{\prime}} \in[0,20] \text { and } \\
\omega_{\nu^{\prime \prime}, k l}=0 \forall\left(\nu^{\prime \prime}, k, l\right) \neq(\nu, i, j),\left(\nu^{\prime}, i^{\prime}, j^{\prime}\right)
\end{array}
\end{array}\right\},
$$



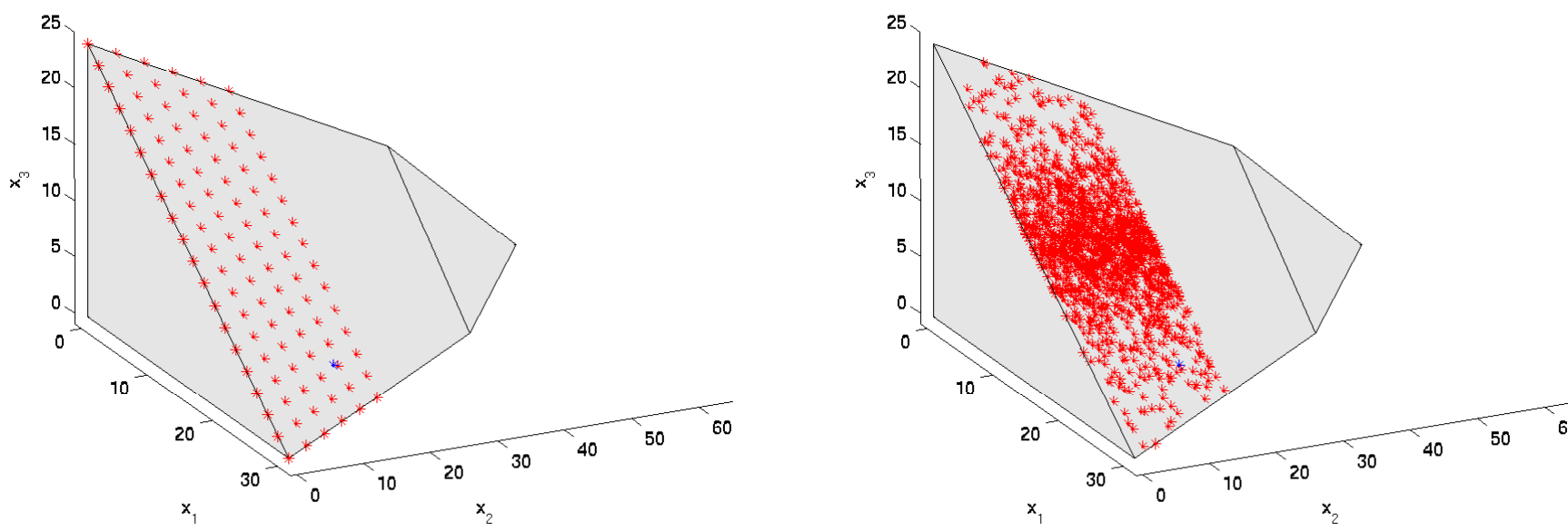

Figure 4: GNEs found by the resource-directed parametrization approach, using grid sampling (left) and random sampling (right), for Example 4.

where $\nu=\sigma((i, j)), \nu^{\prime}=\sigma\left(\left(i^{\prime}, j^{\prime}\right)\right) \in\{1,2\}$ and $(i, j),\left(i^{\prime}, j^{\prime}\right) \in \mathcal{A}$ with $(i, j) \neq\left(i^{\prime}, j^{\prime}\right)$. We thus solve at most $1+12 \cdot 20+60 \cdot(20)^{2}=24241$ LCPs and declare a solution $x^{*}$ to be a GNE if it satisfies (4.2). When sampled on a grid, 12699 LCPs are solved, with 66 of the LCPs yielding GNE, all of which are distinct. Of these, 34 GNEs come from sampling on the line segments, with the remaining 32 GNEs coming from sampling on the boxes. Run time is around 49 seconds. When sampled randomly, the number of GNEs found tends to be higher, around 80, all of which are distinct. Run time remains around 49 seconds.

\begin{tabular}{c|ccc} 
Table 3: $\left(\theta_{1}\left(x^{*}\right), \theta_{2}\left(x^{*}\right)\right)$ vs. $\left(\theta_{1}(\bar{x}), \theta_{2}(\bar{x})\right)$ \\
\hline & $\theta_{2}\left(x^{*}\right)>_{\varepsilon} \theta_{2}(\bar{x})$ & $\theta_{2}\left(x^{*}\right)={ }_{\varepsilon} \theta_{2}(\bar{x})$ & $\theta_{2}\left(x^{*}\right)<_{\varepsilon} \theta_{2}(\bar{x})$ \\
\hline$\theta_{1}\left(x^{*}\right)>{ }_{\varepsilon} \theta_{1}(\bar{x})$ & 0 & 20 & 0 \\
$\theta_{1}\left(x^{*}\right)={ }_{\varepsilon} \theta_{1}(\bar{x})$ & 0 & 1 & 0 \\
$\theta_{1}\left(x^{*}\right)<_{\varepsilon} \theta_{1}(\bar{x})$ & 0 & 45 & 0 \\
\hline
\end{tabular}

For each computed GNE $x^{*}$, the objective value pair $\left(\theta_{1}\left(x^{*}\right), \theta_{2}\left(x^{*}\right)\right)$ is compared with the pair $\left(\theta_{1}(\bar{x}), \theta_{2}(\bar{x})\right)$, where $\bar{x}$ is the GNE obtained by solving $\operatorname{VI}(F, X)$ and $\theta_{\nu}(x)=\sum_{i \in \mathcal{N}} \sum_{j \in \mathcal{N}}\left(c_{\nu, i}-p_{j}\left(S_{j}\right)\right) x_{\nu, i j}+\sum_{(i, j) \in \mathcal{A}} e_{i j} x_{\nu, i j}$. Table 3 shows the numbers of GNEs $x^{*}$ that satisfy the respective relations, where $a>_{\varepsilon} b, a={ }_{\varepsilon} b$ and $a<_{\varepsilon} b$ mean $a>$ $b+\varepsilon,|a-b| \leq \varepsilon$ and $a<b-\varepsilon$, respectively. We set $\varepsilon$ to be $10^{-5}$. We see that, for 45 of the 66 GNEs found, firm 1 does better and firm 2 does no worse compared to the GNE $\bar{x}$.

It is instructive to compare in more detail the GNE $\bar{x}$, which is a normalized equilibrium, with one of the other GNEs found. Take the GNE $x^{*}$ found by solving $\operatorname{VI}\left(F^{\omega}, X\right)$ with $\omega_{1,31}=2$ and $\omega_{\nu, i j}=0$ for all $\nu$ and $(i, j) \neq(3,1)$. The sales and the nodal prices of GNEs $\bar{x}$ and $x^{*}$ are summarized in Tables 4 and 5, respectively. (Only the nonzero 
sales are shown.) We observe that the nodal prices in Table 5 are identical for the two GNEs. However, comparing the firms' profit (negated), we have

$$
\begin{aligned}
& \theta_{1}(\bar{x})=-1969.5>-1971.5=\theta_{1}\left(x^{*}\right) \\
& \theta_{2}(\bar{x})=-1923.6=-1923.6=\theta_{2}\left(x^{*}\right) .
\end{aligned}
$$

Thus, $\bar{x}$ is weakly dominated by $x^{*}$ in the sense that, at the GNE $\bar{x}$, firm 1 may be motivated to move to $x^{*}$ by paying a small incentive $\varepsilon>0$ to firm 2 . (Recall that each player is maximizing its profit.) Hence, the GNE $\bar{x}$ is unstable and less likely to arise in reality. This shows that the approaches in $[6,7,32]$ may fail to find some important GNEs, while the proposed parametrization approach has a better chance to find those GNEs, though at extra computational cost.

Table 4: Firms' sales

\begin{tabular}{ccccccccc}
\hline$(\nu, i, j)$ & $(1,1,1)$ & $(1,1,3)$ & $(1,2,2)$ & $(1,2,3)$ & $(2,2,1)$ & $(2,2,2)$ & $(2,3,1)$ & $(2,3,3)$ \\
\hline $\bar{x}_{\nu, i j}$ & 77.01 & 22.99 & 41.84 & 8.16 & 59.83 & 40.17 & 2.85 & 47.15 \\
$x_{\nu, i j}^{*}$ & 78.01 & 21.99 & 41.84 & 8.16 & 59.83 & 40.17 & 1.85 & 48.15 \\
\hline
\end{tabular}

Table 5: Nodal prices

\begin{tabular}{cccc}
\hline node $i$ & 1 & 2 & 3 \\
\hline$p_{i}\left(S_{i}\right)$ & 28.82 & 27.82 & 27.82 \\
$p_{i}\left(S_{i}^{*}\right)$ & 28.82 & 27.82 & 27.82 \\
\hline
\end{tabular}

For the resource-directed parametrization approach, we sample $N_{s}=5$ points per interval $[0,1]$, with $\rho=\infty$. For simplicity, we omit showing $g_{\nu,(i, j)}$ for $\nu=1,2$ and $(i, j) \in \mathcal{A}$ and $X^{\beta}$. Here $\beta^{\min }=(-16.125,-12.5,-10,-10,-8.5,-12.125)^{T}$. We solve $5^{6}=15625$ LCPs and declare a solution $x^{*}$ to be a GNE if it satisfies (4.2). However, this yielded no GNE when sampled on a grid or randomly! We speculate that, for this example, the GNEs lie mainly in low-dimensional faces of $X$.

\section{Concluding Remarks}

We have proposed and analyzed two parametrized VI approaches for finding a "representative" set of GNEs, and have applied them to three examples from the literature by sampling over the parameter spaces. Our analysis and numerical results suggest that the proposed approaches can find important GNEs that elude existing approaches $[6,7,32]$. In our numerical tests, the price-directed parametrization generally achieved a higher GNE yield rate. On the other hand, for Example 4, the resource-directed parametrization found GNEs that are spatially more evenly distributed. The two approaches appear to complement each other, with one tending to find GNEs lying in low-dimensional faces of the feasible set $X$ and the other tending to find GNEs lying in high-dimensional faces of $X$. 
In the price-directed parametrization, we have to restrict the parameter space heuristically because Theorem 3.4 does not say how large the parameter space should be. Moreover, in our numerical tests, we sampled the parameters either randomly or on a grid from a box in the parameter space. While such simple procedures work reasonably well for small GNEP, more efficient parameter search procedures are likely needed to handle larger GNEPs, as is discussed in Subsection 3.4. These are topics for future study.

\section{References}

[1] Arrow, K.J., Debreu, G.: Existence of an equilibrium for a competitive economy, Econometrica 22, 265-290 (1954)

[2] Bertsekas, D.P. (in collaboration with Nedić, A., Ozdaglar, A.E.): Convex Analysis and Optimization. Athena Scientific, Belmont, (2003)

[3] Bremner, D., Fukuda, K., Marzetta, A.: Primal-dual methods for vertex and facet enumeration. Discrete Comput. Geom. 20, 333-357 (1998)

[4] Contreras, J., Klusch, M., Krawczyk, J.B.: Numerical solutions to Nash-Cournot equilibria in coupled constraint electricity markets. IEEE Trans. Power Syst. 19, 195-206 (2004)

[5] Dontchev, A.L., Rockafellar, R.T.: Robinson's implicit function theorem and its extensions. Math. Program. 117, 129-147 (2008)

[6] Facchinei, F., Fischer, A., Piccialli, V.: On generalized Nash games and variational inequalities. Oper. Res. Lett. 35, 159-164 (2007)

[7] Facchinei, F., Fischer, A., Piccialli, V.: Generalized Nash equilibrium problems and Newton methods. Math. Program. 117, 163-194 (2008)

[8] Facchinei, F., Kanzow, C.: Generalized Nash equilibrium problems. 4OR 5, 173$210(2007)$

[9] Facchinei, F., Pang, J.-S.: Finite-Dimensional Variational Inequalities and Complementarity Problems. Springer-Verlag, New York (2003)

[10] Facchinei, F., Pang, J.-S.: Exact penalty functions for generalized Nash problems. In: Di Pillo, G., Roma, M. (eds.) Large-Scale Nonlinear Optimization, pp. 115-126 Springer, Heidelberg (2006)

[11] Ferris, M.C., Munson, T.S.: Complementarity problems in GAMS and the PATH solver. J. Econ. Dyn. Control 24, 165-188 [ftp://ftp.cs.wisc.edu/mathprog/solvers/path/matlab] (2000)

[12] Fukushima, M.: Restricted generalized Nash equilibria and controlled penalty algorithm. Technical Report 2008-007, Department of Applied Mathematics and Physics, Graduate School of Informatics, Kyoto University (July 2008) 
[13] Harker, P.T.: Generalized Nash games and quasi-variational inequalities. Eur. J. Oper. Res. 54, 81-94 (1991)

[14] Harker, P.T., Pang, J.-S.: Finite-dimensional variational inequality and nonlinear complementarity problems: A survey of theory, algorithms and applications. Math. Program. 48, 161-220 (1990)

[15] von Heusinger, A., Kanzow, C.: Optimization Reformulations of the Generalized Nash Equilibrium problem using Nikaido-Isoda-type functions. Comput. Optim. Appl. (to appear), DOI 10.1007/s10589-007-9145-6.

[16] Hobbs, B.F.: Linear complementarity models of Nash-Cournot competition in bilateral and POOLCO power market. IEEE Trans. Power Syst. 16, 194-202 (2001)

[17] Janin, R.: Directional derivative of the marginal function in nonlinear programming. Math. Programming Stud. 21, 110-126 (1984)

[18] Josephy, N.H.: Newton's method for generalized equations and the PIES energy model. Ph.D. Dissertation, Department of Industrial Engineering, University of Wisconsin-Madison (May 1979)

[19] Krawczyk, J.B., Uryasev, S.: Relaxation algorithms to find Nash equilibria with economic applications. Environ. Model. Assess. 5, 63-73 (2000)

[20] Krawczyk, J.B.: Numerical solutions to coupled-constraint (or generalized Nash) equilibrium problems. Comput. Manag. Sci. 4, 183-204 (2007)

[21] Luo, Z.Q., Pang, J.-S., Ralph, D.: Mathematical Programs with Equilibrium Constraints. Cambridge University Press, Cambridge (1996)

[22] Nikaido, H., Isoda, K.: Note on noncooperative convex games. Pac. J. Math. 5, 807-815 (1955)

[23] Outrata, J.V., Zowe, Z.: A Newton method for a class of quasi-variational inequalities. Comput. Optim. Appl. 4, 5-21 (1995)

[24] Pang, J.-S., Fukushima, M.: Quasi-variational inequalities, generalized Nash equilibria, and multi-leader-follower games. Comput. Manag. Sci. 2, 21-56; Erratum. Comput. Manag. Sci. (to appear), DOI 10.1007/s10287-009-0093-8.

[25] Robinson, S.M.: Shadow prices for measures of effectiveness, I: Linear model. Oper. Res. 41, 518-535 (1993)

[26] Robinson, S.M.: Shadow prices for measures of effectiveness, II: General model. Oper. Res. 41, 536-548 (1993)

[27] Robinson, S.M.: Solution continuity in monotone affine variational inequalities. SIAM J. Optim. 18, 1046-1060 (2007)

[28] Rockafellar, R.T.: Lagrange multipliers and optimality. SIAM Rev. 35, 183-238 (1993) 
[29] Rosen, J.B.: Existence and uniqueness of equilibrium points for concave $N$-person games. Econometrica 33, 520-534 (1965)

[30] Uryasev, S., Rubinstein, R.Y.: On relaxation algorithms in computation of noncooperative equilibria. IEEE Trans. Autom. Control 39, 1263-1267 (1994)

[31] Varian, H.R.: Microeconomic Analysis. W. W. Norton Co., New York (1992)

[32] Wei, J.-Y., Smeers, Y.: Spatial oligopolistic electricity models with Cournot generators and regulated transmission prices. Oper. Res. 47, 102-112 (1999) 